\title{
REGULARITY OF OPTIMAL SHAPES FOR THE DIRICHLET'S ENERGY WITH VOLUME CONSTRAINT*
}

\author{
TANGUY BRIANCON ${ }^{1,2}$
}

\begin{abstract}
In this paper, we prove some regularity results for the boundary of an open subset of $\mathbb{R}^{d}$ which minimizes the Dirichlet's energy among all open subsets with prescribed volume. In particular we show that, when the volume constraint is "saturated", the reduced boundary of the optimal shape (and even the whole boundary in dimension 2) is regular if the state function is nonnegative.
\end{abstract}

Mathematics Subject Classification. 35R35, 49N60, 49Q10.

Received 25 February 2003. Revised 4 August 2003.

\section{INTRODUCTION}

In this paper, we study the regularity of the boundary of an open subset of $\mathbb{R}^{d}$ which minimizes Dirichlet's energy among all open subsets with prescribed Lebesgue's measure and included in a fixed open subset of $\mathbb{R}^{d}$.

More precisely, let $D \subset \mathbb{R}^{d}$ be open, $f \in L^{2}(D) \cap L^{\infty}(D)$ and $a>0$. For each $\Omega \subset D$ open with $|\Omega|=a$ (where $|\Omega|$ is the Lebesgue's measure of $\Omega$ ), we define $u_{\Omega}$ as the solution of,

$$
u_{\Omega} \in \mathrm{H}_{0}^{1}(\Omega),-\Delta u_{\Omega}=f \text { on } \Omega .
$$

We know that $u_{\Omega}$ minimizes the Dirichlet energy,

$$
J(u)=\int_{D} \frac{1}{2}|\nabla u|^{2} \mathrm{~d} x-\int_{D} f u,
$$

among all $u \in \mathrm{H}_{0}^{1}(\Omega)$ (recall that $\mathrm{H}_{0}^{1}(\Omega) \subset \mathrm{H}_{0}^{1}(D)$ ). Our goal is to study the regularity of $\partial \Omega^{*}$ where $\Omega^{*}$ is a solution of:

$$
J\left(u_{\Omega^{*}}\right)=\min \left\{J\left(u_{\Omega}\right), \Omega \subset D \text { open },|\Omega|=a\right\} .
$$

This problem has been studied, for example, in $[5,14]$ and in [11]. We mainly prove that, when the constraint $\left|\Omega^{*}\right|=a$ is "saturated", then the reduced boundary $\partial^{*} \Omega$ is regular in regions where $u$ does not change its sign

Keywords and phrases. Shape optimization, calculus of variations, free boundary, geometrical measure theory.

* This work was made during my Ph.D. thesis at the University of Rennes 1 and at the École Normale Supérieure de Cachan in Rennes. I would like to thank very much my advisor, Michel PIERRE, for his help. I also thank the referee for his helpful remarks.

1 Université Rennes 1.

2 Antenne de Bretagne de l'École Normale Supérieure de Cachan; e-mail: briancon@bretagne.ens-cachan.fr 
(see e.g. $[7,9]$ or $[6]$ for a definition of $\partial^{*} \Omega^{*}$ ). Moreover we prove that, in dimension 2 , the full boundary $\partial \Omega^{*}$ is regular if $u$ is nonnegative.

Let us make some comments on the result. Recall that, if $\partial \Omega^{*}$ is regular, the Euler-Lagrange equation of the problem (1) may be written as:

$$
\left\{\begin{array}{rrrr}
(a)-\Delta u^{*} & = & f \text { in } \Omega^{*}, \\
(b) & u^{*} & = & 0 \text { on } \partial \Omega^{*}, \\
(c) & \left|\nabla u^{*}\right| & = & \Lambda \text { on } \partial \Omega^{*} .
\end{array}\right.
$$

This free boundary system appears in several places in the literature. Regularity for the corresponding various minimization problems has been also considered. Our approach is widely inspired by those in [2,3] or [10]. In these papers, penalized version of (1) are considered like, for instance, minimizing expressions of the kind

$$
J\left(u_{\Omega}\right)+\lambda|\Omega|
$$

Actually, we prove that our constraint problem may be also written, in the saturated case, as a "pseudopenalized" problem in the following way: the solution $u^{*}=u_{\Omega^{*}}$ satisfies:

i) $\exists \lambda^{*}>0$ such that $J\left(u^{*}\right)+\lambda^{*}\left|\Omega^{*}\right| \leq J(v)+\lambda^{*}|\{v \neq 0\}|$,

for all $v$ such that $\left|\Omega^{*}\right| \leq|\{v \neq 0\}|$;

ii) $\exists \mu(h)>0$ such that $J\left(u^{*}\right)+\mu(h)\left|\Omega^{*}\right| \leq J(v)+\mu(h)|\{v \neq 0\}|$,

for all $v$ such that $\left|\Omega^{*}\right|-h \leq|\{v \neq 0\}| \leq\left|\Omega^{*}\right|$.

Then we are able to prove a weaker version of the Euler-Lagrange's equation (2), namely

$$
\Delta u_{\Omega^{*}}+f \chi_{\Omega^{*}}=\Lambda \mathcal{H}^{d-1}\left\lfloor\partial \Omega^{*}\right.
$$

in the sense of distribution in $D$, where $\mathcal{H}^{d-1}\left\lfloor\partial \Omega^{*}\right.$ is the restriction to $\partial \Omega^{*}$ of the $d-1$ dimensional Hausdorff measure and $\chi_{\Omega^{*}}$ is the characteristic function of $\Omega^{*}$. For doing this, and in particular for the existence of a positive $\mu(h)$, we need one hypothesis saying that the constraint is saturated (see Rem. 1.4). As proved in the last section, this hypothesis is always true if $f \geq 0, f \not \equiv 0$. Note that we do not need this hypothesis for the existence of $\lambda^{*}$.

Our goal is to prove that, nevertheless, after some work, the regularity question may fit into the approach in $[2,10]$, at least in the saturated case. After reaching this main step, we are able to directly use regularity results proved in $[2,10]$ for what they called weak solutions. We deduce that, in regions where the sign of $u_{\Omega^{*}}$ does not change, then $\partial^{*} \Omega^{*}$ is regular. We also reach full regularity of $\partial \Omega^{*}$ in dimension 2 in the positive case. Note that cusps may occur for the boundary at points where $u$ changes its sign, even in dimension 2 .

Although different, this may be compared with [1] where pure constraints are also considered and also reduced to a "penalized" version. But our problem and our apprach are different. First, here the nonhomogeneity is inside the domain and not on the boundary. This makes the situation slightly different. For instance, an assumption of "saturation" is necessary to obtain regularity. Next, in [1], the authors first deduce the regularity of the boundary for penalized problem from the results in [2]. Then, they use this regularity to prove that it is equivalent to the problem with volume constraint. Here we prove directly the equivalence between constrained and penalized problems. This is valid without sign condition and without a priori knowledge of regularity.

Our paper is organized as follows.

In Section 1, we recall the known existence and regularity results for our problem and we state our main results. We know that one first step in proving regularity of the boundary $\partial \Omega^{*}$ is to prove regularity of the solution $u_{\Omega^{*}}$, for instance that $u_{\Omega^{*}}$ is Lipschitz continuous when $u_{\Omega^{*}} \geq 0$. This will be assumed here and references will be given, in particular [4], [13] or also [2,10]. 
We will show in Section 2 that $\Delta u_{\Omega^{*}}+f \chi_{\Omega^{*}}$ is in $D$ the difference of two Radon measures absolutely continuous with respect to $\mathcal{H}^{d-1}\left\lfloor\partial \Omega^{*}\right.$ (which is not yet, at this step, a Radon measure). This may be seen as the easier half of (5). Moreover we will see (under some hypothesis, see Th. 2.4), that $\Omega^{*}$ has (locally) finite perimeter (for the definitions and properties of sets with finite perimeter see for example $[6,9]$ or $[7]$ ).

In Section 3 we study the blow-up of $u_{\Omega^{*}}$ (i.e. limits of $u_{\Omega^{*}}\left(x_{0}+r x\right) / r$ as $r$ goes to 0$)$ and in particular around $x_{0} \in \partial^{*} \Omega^{*}$ where we can identify the limit function.

In Section 4, we want to control $J\left(u_{\Omega}\right)-J\left(u_{\Omega^{*}}\right)$ in term of $|\Omega|-\left|\Omega^{*}\right|$, with $|\Omega|$ close to $a$. We essentially show (3) and (4). Moreover if we define, for $h>0, \mu(h)$ as the biggest $\mu$ such that (4) is true for every $\Omega$ with $\left|\Omega^{*}\right|-h \leq|\Omega| \leq\left|\Omega^{*}\right|$ and $\lambda^{*}(h)$ as the smallest $\lambda^{*}$ such that (3) is true for all $\Omega$ with $|\Omega| \leq\left|\Omega^{*}\right|+h$, then we prove that $\lim _{h \rightarrow 0} \mu(h)=\lim _{h \rightarrow 0} \lambda^{*}(h)$. This proves that "asymptotically", our problem is equivalent to a penalized version with this common limit.

This will allow us, in Section 5, to use some methods from [2] and [10], under the hypothesis $u \geq 0$. First we show that $\mathcal{H}^{d-1}\left(\partial \Omega^{*} \backslash \partial^{*} \Omega^{*}\right)=0$, then the absolute continuity of $\mathcal{H}^{d-1}\left\lfloor\partial \Omega^{*}\right.$ (now a Radon measure) with respect to $\Delta u_{\Omega^{*}}+f \chi_{\Omega^{*}}$. Finally, with the result of blow-up near $\partial^{*} \Omega^{*}$, we are able to derive their exact relationship: it is exactly given by (5). With this, we get that $u_{\Omega^{*}}$ is a weak solution in the sense of [3] and [10]: so we may deduce the $\mathrm{C}^{1}$ regularity of $\partial^{*} \Omega^{*}$. Moreover, using the precise behavior of $\mu(h)$ and $\lambda^{*}$, we get (as in [3]) the regularity of the whole boundary $\partial \Omega^{*}$ in dimension 2.

Finally, in Section 6, we discuss the hypothesis of saturation. We show that this hypothesis is always true if $f \geq 0, f \neq 0$. After that we also remark that this hypothesis is true for, at least, a dense open subset of $a$.

\section{The MAIN RESUlts}

\subsection{Existence, first results}

We recall here the necessary existence results for our problem.

Let $D$ be any open subset of $\mathbb{R}^{d}, f \in L^{2}(D) \cap L^{\infty}(D)$ and $0<a<|D|$ (we denote by $|E|$ the Lebesgue's measure of any measurable subset $E$ of $\left.\mathbb{R}^{d}\right)$. For every $u \in \mathrm{H}_{0}^{1}(D)$, we define

$$
J(u)=\int_{D} \frac{1}{2}|\nabla u|^{2} \mathrm{~d} x-\int_{D} f u,
$$

and $\Omega_{u}=\{x \in D ; u(x) \neq 0\}=\{u \neq 0\}$. We are interested in the regularity of the solution of the following shape optimization problem:

$$
\left(\mathcal{P}_{f}\right) J\left(u_{\Omega^{*}}\right)=\inf \left\{J\left(u_{\Omega}\right), \Omega \subset D \text { open },|\Omega|=a\right\}
$$

where $u_{\Omega}$ is defined by:

so that $u_{\Omega}$ is the solution of:

$$
J\left(u_{\Omega}\right)=\min \left\{J(v), v \in \mathrm{H}_{0}^{1}(\Omega)\right\}
$$

$$
-\Delta u_{\Omega}=f, u \in \mathrm{H}_{0}^{1}(\Omega)
$$

It is easy to see that, if there exists $w \in \mathrm{H}_{0}^{1}(D)$ such that $-\Delta w=f$ in $\mathrm{D}$ with $\left|\Omega_{w}\right|<a$, then any open set $\Omega^{*}$ such that $\Omega_{w} \subset \Omega^{*} \subset D$ and $\left|\Omega_{w}\right|=a$ is a solution of $\left(\mathcal{P}_{f}\right)$. Similarly, if there exists $w \in \mathrm{H}_{0}^{1}(D)$ such that $-\Delta w=f$ in $\mathrm{D}$ and $\left|\Omega_{w}\right|=a$, then $\Omega^{*}=\Omega_{w}$ is the only possible solution of $\left(\mathcal{P}_{f}\right)$. As proved in $[11,12] \Omega_{w}$, may even not be open! In fact, one can expect regularity of $\Omega^{*}$ only if the volume constraint $\left|\Omega_{w}\right| \leq a$ is effective. Therefore, since we are interested in proving regularity, in the rest of this paper, we will naturally assume that:

$$
\left.\begin{array}{c}
\text { There does not exist any } v \in \mathrm{H}_{0}^{1}(D) \text { with }\left|\Omega_{v}\right| \leq a \\
\text { such that }-\Delta v=f \text { in } D .
\end{array}\right\}
$$

It turns out that this condition implies (see [5]) that the following problem has a solution, 
where $\mathbb{V}_{a}^{0}$ is defined by

$$
(\mathcal{P})\left\{\begin{array}{r}
u \in \mathrm{H}_{0}^{1}(D),\left|\Omega_{u}\right|=a \\
J(u) \leq J(v), \forall v \in \mathbb{V}_{a}^{0},
\end{array}\right.
$$

$$
\mathbb{V}_{a}^{0}=\left\{v \in \mathrm{H}_{0}^{1}(D),\left|\Omega_{v}\right| \leq a\right\}
$$

And it is clear that, if a solution $u$ of $(\mathcal{P})$ is continuous in $D$, then the open set $\Omega_{u}$ is a solution of the shape optimization problem $\left(\mathcal{P}_{f}\right)$. Using $(7)$, we see that, if $u \in \mathbb{V}_{a}^{0}$ is such that $J(u) \leq J(v)$ for all $v \in \mathbb{V}_{a}^{0}$ then, $\left|\Omega_{u}\right|=a$ and $u$ is a solution of $(\mathcal{P})$.

Remark 1.1. If $f \geq 0, f \neq 0$ by the (strict) maximum principle (7) holds. Moreover if $u$ is a solution of $(\mathcal{P})$ then $u \geq 0$.

In the following we will only consider solutions of $(\mathcal{P})$.

As explained above; to obtain regularity, we will have to assume that the volume constraint $\left|\Omega_{u}\right|=a$ (or $\left|\Omega_{u}\right| \leq a$ ) does play its role. Part of this is contained in assumption (5). But we will also assume that the Lagrange- multiplier $\lambda$ in the Euler-Lagrange's equation of the minimization problem is strictly positive.

Proposition 1.2 (Euler Lagrange's equation). Let $u$ be a solution of $(\mathcal{P})$. Then there exists $\lambda \geq 0$ such that for all $\Phi \in \mathrm{C}_{0}^{\infty}\left(D, \mathbb{R}^{d}\right)$ we have:

$$
\int_{D}\langle D \Phi \nabla u \cdot \nabla u\rangle-\frac{1}{2} \int_{D}|\nabla u|^{2} \operatorname{div} \Phi=\int_{D} f\langle\nabla u \cdot \Phi\rangle+\lambda \int_{\Omega_{u}} \operatorname{div} \Phi .
$$

Remark 1.3. This is proved in [5]. The idea is to write that the derivative of $t \rightarrow J(u(I+t \Phi))$ vanishes at $t=0$ for $\Phi$ satisfying $\int_{\Omega_{u}} \operatorname{div} \Phi \geq 0$. Then, the Lagrange multiplier $\lambda$ appears for general $\Phi$.

Notation: In the rest of this paper, we will be mainly interested in solutions of $(\mathcal{P})$ verifying the Euler-Lagrange equation in $D$ with $\lambda>0$ : we will simply write that $u$ is a solution of $(\mathcal{P})$ with $\lambda>0$.

Remark 1.4. As explained above, if the constraint $\left|\Omega_{u}\right| \leq a$ is not "saturated" (i.e. $\lambda=0$ ) the optimal form may not be regular. For instance, we can construct a solution $u$ of $(\mathcal{P})$ with $\lambda=0$ and $\partial \Omega_{u}$ very unregular as follows: take $D=B(0,1), u \in \mathrm{C}_{0}^{\infty}(D)$, such that $\partial \Omega_{u}$ is not regular, $f=-\Delta u$ and $a=\left|\Omega_{u}\right|$. It is obvious that $u$ is a solution of $(\mathcal{P})$ since $J(u) \leq J(v)$ for all $v \in \mathrm{H}_{0}^{1}(D)$. In Section 6 we will discuss the saturation hypothesis $\lambda>0$. We show that if $f \geq 0, f \not \equiv 0$ this is always true. If $f$ changes its sign, this happen at least for a dense open subset of $a$.

\subsection{The regularity result}

Our main regularity result is the following. As usual, if $\Omega$ has finite perimeter, we denote by $\partial^{*} \Omega$ the reduced boundary of $\Omega$, and by $\mathcal{H}^{d-1}$ the Hausdorff measure of dimension $d-1$. (see $[6,9]$ or [7]).

Theorem 1.5. Let $u$ be a nonnegative solution of $(\mathcal{P})$ with $\lambda>0$. Then $\Omega_{u}$ has locally finite perimeter in $D$ and,

$$
u \text { is locally Lipschitz continuous in D }
$$

$$
\text { for every compact } K \subset D, \mathcal{H}^{d-1}\left(\left(\partial \Omega_{u} \backslash \partial^{*} \Omega_{u}\right) \cap K\right)=0 \text {. }
$$

Moreover:

$$
\begin{gathered}
\Delta u+f \chi_{\Omega_{u}}=\sqrt{2 \lambda} \mathcal{H}^{d-1}\left\lfloor\partial \Omega_{u}, \text { in } \mathcal{D}^{\prime}(D),\right. \\
\partial^{*} \Omega_{u} \cap D \text { is a } C^{1, \alpha} \text { hypersurface with } \alpha>0 .
\end{gathered}
$$


Finally, if $d=2$, we have $\partial^{*} \Omega_{u} \cap D=\partial \Omega_{u} \cap D$ and so $\partial \Omega_{u}$ is regular in $D$. If $f$ is analytic, $\partial^{*} \Omega_{u}$ (or $\partial \Omega_{u}$ if $d=2$ ) is analytic.

Notation: Here $\mathcal{H}^{d-1}\left\lfloor\partial \Omega_{u}\right.$ denotes the restriction of the measure $\mathcal{H}^{d-1}$ to $\partial \Omega_{u}$.

Extensions: In the case where the sign of $u$ changes in $D$, we also have the same kind of regularity in open subsets of $D$ where the sign of $u$ is constant (see Th. 5.1). This means that, in dimension 2, we can have singularities for $\partial \Omega_{u}$ only in points where $u$ changes its sign (and we know by simple examples that this indeed happens!).

For the proof of this theorem, we use tools from $[2,10]$. In these papers, the authors study the regularity of minima of functionals like:

$$
G(u)=\int_{D_{1}} \frac{1}{2}|\nabla u|^{2} \mathrm{~d} x-\int_{D_{1}} f u+\lambda|\{u>0\}|,
$$

with $u \geq 0, u=u_{0}$ on $\partial D_{1}(f=0$ in [2]). The above problem looks like "penalized" versions of our problem. Indeed, in [2] and in [10] there is no constraint such as $\left|\Omega_{u}\right| \leq a$, but there is the extra term " $\lambda|\{u>0\}|^{\prime \prime}$ in the functional. This may be viewed as a penalization term for our problem.

Our strategy (and main task) will actually consist in showing that, for our problem, there are, in general, two constants $\mu$ and $\lambda^{*}$ with $0<\mu \leq \lambda \leq \lambda^{*}$ such that (4) and (3) hold. Then, using technics from [2,10] we are able to prove (10) and (11).

More precisely, we show the following.

Proposition 1.6. Let $u$ be a solution of $(\mathcal{P})$ with $\lambda>0$ (see Prop. 1.2). Let $B\left(x_{0}, r\right) \subset D$ be such that $\left|B\left(x_{0}, r\right)\right|<|D|-a$,

$$
0<\frac{\left|B\left(x_{0}, r\right) \cap \Omega_{u}\right|}{\omega_{d} r^{d}}<1,
$$

and $u$ not identically 0 on $\partial B\left(x_{0}, r\right)$. Let

$$
\mathcal{F}_{0}=\left\{v \in \mathrm{H}_{0}^{1}(D) ; u-v \in \mathrm{H}_{0}^{1}(B(x, r))\right\} .
$$

For $h>0$ we define

Then

$$
\begin{gathered}
\mu(h)=\sup \left\{\mu \geq 0 ; J(u)+\mu\left|\Omega_{u}\right| \leq J(v)+\mu\left|\Omega_{v}\right|, \forall v \in \mathcal{F}_{0}, a-h \leq\left|\Omega_{v}\right| \leq a\right\}, \\
\lambda^{*}(h)=\inf \left\{\lambda^{*} \geq 0 ; J(u) \leq J(v)+\lambda^{*}\left(\left|\Omega_{v}\right|-a\right)^{+}, \forall v \in \mathcal{F}_{0},\left|\Omega_{v}\right| \leq a+h\right\} .
\end{gathered}
$$

and

$$
\mu(h) \leq \lambda \leq \lambda^{*}(h)<+\infty
$$

$$
\lim _{h \rightarrow 0} \mu(h)=\lim _{h \rightarrow 0} \lambda^{*}(h)=\lambda .
$$

In particular, $\mu(h)>0$ for $h$ small enough.

Remark 1.7. The fact that $\lambda^{*}(h)$ exists and is finite may be found essentially in [13]. It means that:

$$
J(u)+\lambda^{*}(h)\left|\Omega_{u}\right| \leq J(v)+\lambda^{*}(h)\left|\Omega_{v}\right|
$$

for all $v \in \mathcal{F}_{0}$ with $a \leq\left|\Omega_{u}\right| \leq a+h$.

The definition of $\mu(h)$ means that

$$
J(u)+\mu(h)\left|\Omega_{u}\right| \leq J(v)+\mu(h)\left|\Omega_{v}\right|,
$$

for all $v \in \mathcal{F}_{0}$ with $a-h \leq\left|\Omega_{v}\right| \leq\left|\Omega_{u}\right|$, The most important point is that $\mu(h)>0$ (at least for $h$ small enough). The precise value of the limit of $\mu(h)$ as $h \rightarrow 0$ will only be used in dimension 2 . 
If $x_{0} \in \partial^{*} \Omega_{u}$, we have $\lim _{r \rightarrow 0} \frac{\left|B\left(x_{0}, r\right) \cap \Omega_{u}\right|}{\omega_{d} r^{d}}=\frac{1}{2}$, and so, condition (14) is true for $r$ small enough. Moreover, since $x_{0} \in \partial \Omega_{u}$, we can find $r$ such that $u$ is not equal to 0 on $\partial B\left(x_{0}, r\right)$. Therefore, Proposition 1.6 may be applied to all $x_{0} \in \partial \Omega^{*}$.

If we have $\mu(h)=\lambda^{*}(h)$, we get that $u$ is exactly a minimum for (13). But, in general, we have $\mu(h)<\lambda^{*}(h)$ and the two problems are different. For instance, if we take $D=B(0,1)$ in $\mathbb{R}^{2}$ and $f=1$, it is easy to compute exactly $\mu$ and $\lambda$ and to show that $\mu(h)<\lambda^{*}(h)$ (see [4] for details).

The main point is that although the problem is different from those considered in [2] and in [10], we will reach the same kind of regularity for the boundary.

It is well-known that the first step in proving regularity for the boundary of optimal shapes is to prove regularity of the state function. We will not do it here, but rather add it in our assumptions when needed and refer to corresponding previous results in the literature. For instance we have.

Theorem 1.8. Let $u$ be a solution of $(\mathcal{P})$; then $u$ is Hölder-continuous with power $\alpha$ for every $0<\alpha<1$. Moreover, for every open $D_{1}$ such that $D_{1} \subset\{u \geq 0\}$ (or $D_{1} \subset\{u \leq 0\}$ ) u is locally Lipschitz on $D_{1}$. Finally, if $D=\mathbb{R}^{d}$ and $u \geq 0$, $u$ is globally Lipschitz on $\mathbb{R}^{d}$.

The first part of this theorem is in [13] and in [3]. The proof of the second part may be found in [13] or also in the proof of Lemma 2.5 and Corollary 2.6 in [10] (see also [2] 3.2 and 3.3). It is also proved in dimension 2 in [5].

Note also that Lipschitz regularity of the state function $u$ has been proved in [5] in dimension 2 without any positivity assumption and even for solutions of the Euler-Lagrange equation (8).

\section{STUDY OF $\Delta u+f \chi_{\Omega_{u}}$}

In this section, $u$ is a solution of $(\mathcal{P})$.

We show that $\Delta u+f \chi_{\Omega_{u}}$ is the difference of two Radon measure. We start with a technical proposition.

Proposition 2.1. Let $p \in W^{1, \infty}(\mathbb{R}, \mathbb{R})$ with $p(0)=0$. Then we have:

$$
p^{\prime}(u)|\nabla u|^{2}-\operatorname{div}(p(u) \nabla u)-f p(u)=0 \text { in } \mathcal{D}^{\prime}(D) .
$$

Proof. Let $\Psi \in C_{0}^{\infty}(D)$ and $p \in W^{1, \infty}(\mathbb{R}, \mathbb{R})$ with $p(0)=0$. Let:

$$
v_{t}(x)=u(x)+t \Psi(x) p(u(x)) .
$$

We have $v_{t} \in \mathrm{H}_{0}^{1}(D)$. Indeed, $|p(u(x))| \leq\left\|p^{\prime}\right\|_{\infty}|\|| u(x) \mid$, so that $v_{t}$ is in $L^{2}(D)$, and:

$$
|\nabla p(u(x))|=\left|p^{\prime}(u(x)) \nabla u(x)\right| \leq\left\|p^{\prime}\right\|_{\infty} \||\nabla u(x)|,
$$

so $\nabla v_{t} \in L^{2}(D)$. Because $u(x)=0$ imply $v_{t}(x)=0$, we get $\left|\Omega_{v}\right| \leq\left|\Omega_{u}\right|$. By minimality of $u$ we deduce:

$$
\begin{aligned}
0 & \leq \frac{1}{2} \int_{D}\left(\left|\nabla u+t p(u) \nabla \Psi+t \Psi p^{\prime}(u) \nabla u\right|^{2}-|\nabla u|^{2}\right)-\int_{D} f(u+t \Psi p(u)-u) \\
& =\int_{D} t\left\langle p(u) \nabla \Psi+\Psi p^{\prime}(u) \nabla u \cdot \nabla u\right\rangle+t^{2}\left|p(u) \nabla \Psi+\Psi p^{\prime}(u) \nabla u\right|^{2}-t f \Psi p(u) .
\end{aligned}
$$

Dividing by $t>0$ and by $t<0$ and letting $t$ go to 0 , we deduce:

$$
0=\int_{D} \nabla \Psi \cdot p(u) \nabla u+\Psi p^{\prime}(u)|\nabla u|^{2}-f \Psi p(u),
$$

which is Proposition 2.1. 
Theorem 2.2. There exist two positive Radon measures $\mu_{1}$ and $\mu_{2}$ such that:

$$
\mu_{1}=\Delta\left(u^{+}\right)+f \chi_{\{u>0\}}, \mu_{2}=\Delta\left(u^{-}\right)-f \chi_{\{u<0\}} .
$$

Moreover there exists $C=C(f, a)$ such that

$$
\|u\|_{\infty} \leq C
$$

Proof. Let $p_{n}$ be defined by:

$$
p_{n}(r)=\left\{\begin{array}{c}
0 \text { if } r \leq \\
n r \text { if } r \in[0,1 / n] \\
1 \text { if } r \geq
\end{array}\right.
$$

and $q_{n}$ by $q_{n}(r)=\int_{0}^{r} p_{n}(s) \mathrm{d} s$ for $r \geq 0$ and $q_{n}(r)=0$ for $r \leq 0$. Applying Proposition 2.1 and using $p_{n}(u) \nabla u=\nabla q_{n}(u)$ we get:

$$
n|\nabla u|^{2} \chi_{\{0<u<1 / n\}}-\Delta\left(q_{n}(u)\right)-f p_{n}(u)=0 .
$$

When $n$ goes to infinity $p_{n}(u)$ converge in $L_{\text {loc }}^{1}(D)$ to $\chi_{\{u>0\}}$ and $q_{n}(u)$ converge to $u^{+}$in $L^{2}(D)$. So we have in $\mathcal{D}^{\prime}(D)$ :

$$
\lim _{n \rightarrow \infty} \Delta q_{n}(u)+f p_{n}(u)=\Delta\left(u^{+}\right)+f \chi_{\{u>0\}}
$$

Let $\mu_{1}^{n}=n|\nabla u|^{2} \chi_{\{0<u<1 / n\}}$ and $\mu_{1}=\Delta\left(u^{+}\right)+f \chi_{\{u>0\}}$. Let $\Psi \in \mathrm{C}_{0}^{\infty}(D)$; by (17) we have

$$
\begin{aligned}
\int \Psi \mu_{1}^{n} & =\int\left(q_{n}(u) \Delta \Psi+f p_{n}(u) \Psi\right) \\
& \leq\|\Delta \Psi\|_{\infty} \int_{\operatorname{supp} \Psi} u^{+}+\|f\|_{\infty} \mid\|\Psi\|_{\infty}
\end{aligned}
$$

because $p_{n}(u) \leq 1$ and $q_{n}(u) \leq u^{+}$. We deduce that the measures $\mu_{n}$ are uniformly bounded on compact sets and so the limit $\mu_{1}$ of $\mu_{1}^{n}$ in $\mathcal{D}^{\prime}(D)$ is a measure. Moreover, using the uniform bound on compact sets, the limit may be understood weakly in the sense of Radon's measures. We proceed in the same way to get a measure $\mu_{2}$ such that:

Let us show the $L^{\infty}$ estimate. We have:

$$
\Delta\left(u^{-}\right)-f \chi_{\{u<0\}}=\mu_{2}
$$

$$
-\Delta\left(u^{+}\right)=f \chi_{\{u>0\}}-\mu_{1} \leq f \chi_{\{u>0\}} .
$$

Because $\left|\Omega_{u} \cap D\right|<|D|$, there exists an open subset $\omega$ such that $\Omega_{u} \subset \omega \subset D$ and $|\omega|<2\left|\Omega_{u} \cap D\right|$. We can use classical $L^{\infty}$ elliptic estimates (see for example [8] Th. 8.16) to show that:

$$
\left\|u^{+}\right\|_{L^{\infty}} \leq C\left\|f \chi_{u>0}\right\|_{L^{q}(\omega)} \leq C\|f\|_{\infty} a^{1 / q},
$$

with $C=C(d,|\omega|, q)$ and $q>d / 2$. We have the same for $u^{-}$.

We now show that, if $u$ is Lipschitz on an open set $D_{1}$ included in $D$, then $\mu_{1}$ and $\mu_{2}$ are absolutely continuous with respect to the Hausdorff measure $\mathcal{H}^{d-1}$ in $D_{1}$. More precisely we have the following:

Proposition 2.3. Let $u$ be a solution of $(\mathcal{P})$ and $D_{1}$ be an open subset of $D$, such that $u$ is Lipschitz continuous on $D_{1}$. Then there exists $C>0$ such that, for every ball $B(x, r) \subset D_{1}$ with $r \leq 1$, we have:

$$
\begin{aligned}
& \mu_{1}(B(x, r)) \leq C r^{d-1}, \\
& \mu_{2}(B(x, r)) \leq C r^{d-1} .
\end{aligned}
$$


Proof. We use the same notations as in the proof of Theorem 2.2. Let $B(x, r) \subset D_{1}$, then:

$$
\begin{aligned}
\int_{B(x, r)}\left(\Delta q_{n}(u)+f p_{n}(u)\right) & =\int_{\partial B(x, r)} p_{n}(u)\langle\nabla u . n\rangle+\int_{B(x, r)} f p_{n}(u) \\
& \leq\|\nabla u\|_{\infty, D_{1}} \mathrm{~d} \omega_{d} r^{d-1}+\|f\|_{\infty} \omega_{d} r^{d} \\
& \leq C r^{d-1}
\end{aligned}
$$

for $r \leq 1$. Because $\Delta q_{n}(u)+f p_{n}(u)$ converges weakly in the sense of Radon measures to $\Delta u+f \chi_{\{u>0\}}$, we deduce:

$$
\mu_{1}(B(x, r)) \leq \liminf _{n \rightarrow \infty} \int_{B(x, r)}\left(\Delta q_{n}(u)+f p_{n}(u)\right) \leq C r^{d-1} .
$$

We can do the same for $\mu_{2}$.

We will now see that, under some extra hypotheses, $\Omega_{u} \cap D_{1}$ has a finite perimeter on bounded subsets $D_{1}$ of $D$ where $u$ is Lipschitz continuous. We have the following theorem:

Theorem 2.4. Let $u$ be a solution of $(\mathcal{P})$ with $\lambda>0$ and let $D_{1}$ be a bounded open subset of $D$, with $\overline{D_{1}} \subset D$ and where $u$ is Lipschitz continuous. Then $\Omega_{u}=\{|u| \neq 0\}$ has finite perimeter in $D_{1}$. Moreover, there exist constants $C, C^{1}, r_{0}$ depending on the data such that for every $B(x, r) \subset D_{1}$ with $r \leq r_{0}$ :

$$
P\left(\Omega_{u}, B(x, r)\right) \leq C\left(\mu_{1}(B(x, r))+\mu_{2}(B(x, r))\right) \leq C^{1} r^{d-1},
$$

(where $\mu_{1}$ and $\mu_{2}$ are the two measure defined in Th. 2.2).

The proof of Theorem 2.4 will require the following lemma which says in a very weak sense that " $|\nabla u|^{2}=2 \lambda "$ on $\partial\{u \neq 0\}$.

Proposition 2.5. Let $u$ be a solution of $(\mathcal{P})$ and let $D_{1}$ be an open subset of $D$ where $u$ is locally Lipschitz continuous. Then, for every $\varphi \in \mathrm{C}_{0}^{\infty}\left(D_{1}, \mathbb{R}^{d}\right)$ we have:

$$
\lim _{\varepsilon \rightarrow 0} \frac{1}{\varepsilon} \int_{\{0<|u|<\varepsilon\}}\langle\varphi \cdot \nabla|u|\rangle\left(\lambda-\frac{1}{2}|\nabla u|^{2}\right)=0 .
$$

Proof. Let $\varphi \in \mathrm{C}_{0}^{\infty}\left(D_{1}, \mathbb{R}^{d}\right)$ and $\Psi_{\varepsilon}(x)=\max \left(0,1-\frac{|x|}{\varepsilon}\right)$. We write Euler-Lagrange's equation (8) with $\Phi=$ $\varphi \Psi_{\varepsilon}(u) \in W^{1, \infty}(D)$, which has compact support. For this, because $\Phi \in W_{0}^{1, \infty}(D)$ with compact support in $D$, We can approximate $\Phi$ by $\Phi_{n} \in \mathrm{C}_{0}^{\infty}\left(D_{1}\right)$ in $W^{1,1}\left(D_{1}\right)$ with $\nabla \Phi_{n}$ uniformly bounded. The Euler-Lagrange's equation (8) is true also with $\Phi$. We study each term:

$$
\begin{aligned}
\int_{\{u \neq 0\}} \operatorname{div} \Phi & =\int_{\{u \neq 0\}} \Psi_{\varepsilon}(u) \operatorname{div} \varphi+\int_{0<|u|<\varepsilon}\langle\varphi \cdot \nabla u\rangle \Psi_{\varepsilon}^{\prime}(u) \\
& =\int_{\{u \neq 0\}} \Psi_{\varepsilon}(u) \operatorname{div} \varphi-\int_{\{u \neq 0\}} \frac{1}{\varepsilon}\langle\varphi \cdot \nabla|u|\rangle .
\end{aligned}
$$

Since $\Psi_{\varepsilon}(u) \chi_{\{u \neq 0\}}$ converge to 0 a.e when $\varepsilon$ goes to 0 , by dominated convergence, the first term goes to 0 . For the same reason we get:

$$
\lim _{\varepsilon \rightarrow 0} \int_{D} f\langle\nabla u \cdot \Phi\rangle=\lim _{\varepsilon \rightarrow 0} \int_{\{u \neq 0\}} f \Psi_{\varepsilon}(u)\langle\nabla u \cdot \varphi\rangle=0 .
$$




$$
\begin{aligned}
\int_{\{u \neq 0\}} \operatorname{div} \Phi|\nabla u|^{2} & =\int_{\{u \neq 0\}} \Psi_{\varepsilon}(u) \operatorname{div} \varphi|\nabla u|^{2}+\langle\varphi \cdot \nabla u\rangle \Psi_{\varepsilon}^{\prime}(u)|\nabla u|^{2} \\
& =\int_{\{u \neq 0\}} \Psi_{\varepsilon}(u) \operatorname{div} \varphi|\nabla u|^{2}-\frac{1}{\varepsilon} \int_{0<|u|<\varepsilon}\langle\varphi \cdot \nabla|u|\rangle|\nabla u|^{2} .
\end{aligned}
$$

Using $\nabla u \in L^{2}$, the first term goes to 0 . Finally we also have,

$$
\int_{D}\langle D \Phi \nabla u \cdot \nabla u\rangle=\int_{\{u \neq 0\}} \Psi_{\varepsilon}(u)\langle D \phi \nabla u \cdot \nabla u\rangle-\frac{1}{\varepsilon} \int_{\{0<|u|<\varepsilon\}}|\nabla u|^{2}\langle\varphi \cdot \nabla|u|\rangle,
$$

and the first the first term goes to 0. By writing Euler-Lagrange's (8) equation and letting $\varepsilon$ goes to 0, we get the proposition.

Proof of Theorem 2.4. Let $B(x, r) \subset D_{1}$ and $\varphi \in \mathrm{C}_{0}^{\infty}(B(x, r))$. For almost every $s>0$ the boundary of $\{|u|>s\}$ is regular $\left(\mathrm{C}^{1}\right)$, since on the open set $\{u \neq 0\}$ we have $-\Delta u=f$ so that $u$ is $\mathrm{C}^{1}$ and we can use Sard's lemma, which implies that $|\nabla u|>0$ on $\{|u|=s\}$ for almost every $s$. We can now write, using co-area formula (see 3.4.3 in [6]), and Gauss formula:

$$
\begin{aligned}
\frac{1}{\varepsilon} \int_{\{0<|u|<\varepsilon\}}\langle\varphi \cdot \nabla|u|\rangle & =\frac{1}{\varepsilon} \int_{0}^{\varepsilon} \mathrm{d} s \int_{\{|u|=s\}}\left\langle\varphi \cdot \frac{\nabla|u|}{|\nabla u|}\right\rangle \\
=-\frac{1}{\varepsilon} \int_{0}^{\varepsilon} \mathrm{d} s \int_{\{|u|=s\}}\left\langle\varphi \cdot \nu_{s}\right\rangle & =-\frac{1}{\varepsilon} \int_{0}^{\varepsilon} \mathrm{d} s \int_{\{|u|>s\}} \operatorname{div} \varphi
\end{aligned}
$$

(here $\nu_{s}$ is the outward normal to $\{|u|>s\}$ ). We deduce,

$$
\lim _{\varepsilon \rightarrow 0} \frac{1}{\varepsilon} \int_{\{0<|u|<\varepsilon\}}\langle\varphi \cdot \nabla|u|\rangle=\int_{\{|u|>0\}} \operatorname{div} \varphi .
$$

There exist $s_{0}<r$ such that $\varphi \in \mathrm{C}_{0}^{\infty}\left(B\left(x, s_{0}\right)\right)$. If we suppose that $\|\varphi\|_{\infty} \leq 1$, then:

$$
\frac{1}{\varepsilon} \int_{\{0<|u|<\varepsilon\}}\langle\varphi \cdot \nabla|u|\rangle \frac{1}{2}|\nabla u|^{2} \leq \frac{1}{2 \varepsilon}\|\nabla u\|_{\infty} \int_{\{|u|<\varepsilon\} \cap B(x, s)}|\nabla u|^{2},
$$

for every $s_{0}<s<r$. But we saw in the proof of Theorem 2.2 (see (17)) that

$$
\lim _{n \rightarrow \infty} n|\nabla u|^{2} \chi_{\{0<u<1 / n\}}=\mu_{1}
$$

weakly in the sense of Radon's measure, and it is the same on $\{-1 / n<u<0\}$ with $\mu_{2}$. For almost every $s<r$,

$$
\mu_{1}(\partial B(x, s))=\mu_{2}(\partial B(x, s))=0 .
$$

Let such a $s>s_{0}$, we get:

$$
\lim _{n \rightarrow \infty} n \int_{\{|u|<1 / n\} \cap B(x, s)}|\nabla u|^{2}=\mu_{1}(B(x, s))+\mu_{2}(B(x, s)) .
$$

From Proposition 2.5, (18) and (19) with $\varepsilon=1 / n$ and $n \rightarrow \infty$

$$
\begin{aligned}
\lambda \int_{\Omega_{u} \cap B(x, r)} \operatorname{div} \varphi & \leq \frac{\|\nabla u\|_{\infty, D_{1}}}{2}\left(\mu_{1}(B(x, s))+\mu_{2}(B(x, s))\right) \\
& \leq \frac{\|\nabla u\|_{\infty, D_{1}}}{2}\left(\mu_{1}(B(x, r))+\mu_{2}(B(x, r))\right) .
\end{aligned}
$$


That is, by taking the supremum over $\varphi$ :

$$
P\left(\Omega_{u}, B(x, r)\right) \leq \frac{\|\nabla u\|_{\infty, D_{1}}}{2 \lambda}\left(\mu_{1}(B(x, r))+\mu_{2}(B(x, r))\right) .
$$

To prove that $\Omega_{u}$ has finite perimeter, we use that $\mu_{1}$ and $\mu_{2}$ are finite on the bounded set $D_{1}$. Using Proposition 2.3, we get for $B(x, r) \subset D_{1}$ and $r$ small enough:

$$
P\left(\Omega_{u}, B(x, r)\right) \leq C r^{d-1} .
$$

\section{BLOW-UP}

In this section we study the blow up of a solution around a point $x_{0}$ of the boundary of $\Omega_{u}=\{u \neq 0\}$. We will throughout suppose that $u$ is Lipschitz on an open set around $x_{0}$. In particular $\Omega_{u}$ is open. In the last proposition (see Prop. 3.5), we will also assume that $u$ is nonnegative around $x_{0}$.

Notations. Let $u$ be a solution of $(\mathcal{P})$ and let $D_{1}$ be an open subset of $D$ such that $u$ is Lipschitz continuous in $D_{1}$. Let $x_{m} \in \partial \Omega_{u} \cap D_{1}$ go to $x_{0} \in \partial \Omega_{u} \cap D_{1}$ and $r_{m}$ go to 0 with $B\left(x_{m}, r_{m}\right) \subset D_{1}$. We define:

$$
u_{m}(x)=\frac{u\left(x_{m}+r_{m} x\right)}{r_{m}},
$$

and

$$
\Omega_{m}=\left\{x \in \mathbb{R}^{d}, x_{m}+r_{m} x \in \Omega_{u}\right\} .
$$

We will refer to this as the blow-up of $u$ relatively to $B\left(x_{m}, r_{m}\right)$.

Proposition 3.1. There exists $u_{0}$ Lipschitz-continuous and a measurable set $H^{-}$included in $\mathbb{R}^{d}$ with locally finite perimeter such that, up to a subsequence, $u_{m}$ converges to $u_{0}$ uniformly on every compact set, $\nabla u_{m}$ converges to $\nabla u_{0}{ }^{*}$-weakly in $L^{\infty}\left(\mathbb{R}^{d}\right)$ and $\chi_{\Omega_{m}}$ converges to $\chi_{H^{-}}$in $L_{\text {loc }}^{1}\left(\mathbb{R}^{d}\right)$ and almost everywhere. Moreover for almost every $x \notin H^{-}$, we have $u_{0}(x)=0$.

Proof. Let $R>0$, for $m$ large enough we have $B\left(x_{m}, r_{m} R\right) \subset D_{1}$ and $u\left(x_{m}\right)=0$ so that for $x \in B(0, R)$ :

$$
\left|u_{m}(x)\right| \leq\|\nabla u\|_{\infty, D_{1}}|x|,\left|\nabla u_{m}(x)\right| \leq\|\nabla u\|_{\infty, D_{1}} .
$$

So, up to a sub-sequence, $u_{m}$ converges uniformly on $B(0, R)$ to Lipschitz continuous function $u_{0}$ and $\nabla u_{m}$ *-weakly converges in $L^{\infty}\left(\mathbb{R}^{d}\right)$ to $\nabla u_{0}$.

$$
P\left(\Omega_{m}, B(0, R)\right)=\frac{1}{r_{m}^{d-1}} P\left(\Omega_{u}, B\left(x_{m}, r_{m} R\right)\right) \leq C R^{d-1} .
$$

This implies that that $\chi_{\Omega_{m}}$ is relatively compact in $L_{\text {loc }}^{1}\left(\mathbb{R}^{d}\right)$. So there exists $H^{-}$with locally finite perimeter such that, up to a sub-sequence, $\chi_{\Omega_{m}}$ converge to $\chi_{H^{-}}$in $L_{\text {loc }}^{1}\left(\mathbb{R}^{d}\right)$ and almost-everywhere. For almost every $x \notin H^{-}$, we have:

$$
0=\chi_{H^{-}}(x)=\lim _{m \rightarrow \infty} \chi_{\Omega_{m}}(x),
$$

and for $m$ large enough $x_{m}+r_{m} x \notin \Omega_{u}$, so that $u_{m}(x)=0$.

Proposition 3.2. Let $u_{m}$ be as above. Then, up to a subsequence, $\nabla u_{m}$ converges in $L_{\mathrm{loc}}^{p}\left(\mathbb{R}^{d}\right)$ to $\nabla u_{0}$ for all $1 \leq p<\infty$ and almost everywhere. 
Proof. Let $R>0$. For $m$ large enough so that $B\left(x_{m}, r_{m} R\right) \subset D_{1}$, we have in the sense of distributions:

$$
\Delta u_{m}(x)=r_{m} \Delta u\left(x_{m}+r_{m} x\right)
$$

According to Theorem 2.2, $\Delta u=-f \chi_{\Omega_{u}}+\mu_{1}-\mu_{2}$ with $\mu_{1}$ and $\mu_{2}$ positive measures. We get

$$
\begin{aligned}
\int_{B(0, R)}\left|\Delta u_{m}\right| & =r_{m}^{1-d} \int_{B\left(0, r_{m} R\right)}|\Delta u| \\
& \leq \omega_{d} r_{m} R^{d}\|f\|_{\infty}+r_{m}^{1-d} \mu_{1}\left(B\left(0, r_{m} R\right)\right)+r_{m}^{1-d} \mu_{2}\left(B\left(0, r_{m} R\right)\right) \\
& \leq C R^{d}+C R^{d-1}
\end{aligned}
$$

according to Proposition 2.3. The measures $\left|\Delta u_{m}\right|$ are locally bounded uniformly and, up to a sub-sequence, we deduce the convergence of $\nabla u_{m}$ to $\nabla u_{0}$ in $L_{\text {loc }}^{1}\left(\mathbb{R}^{d}\right)$ and so (up to a sub-sequence) the convergence almosteverywhere. Finally, using the convergence a.e and the uniformly bound on $\left\|\nabla u_{m}\right\|_{\infty}$ we deduce that $\nabla u_{m}$ converges to $\nabla u_{0}$ in $L_{\text {loc }}^{p}\left(\mathbb{R}^{d}\right)$ for all $1 \leq p<+\infty$.

Theorem 3.3 (Euler's equation of $\left.u_{0}\right)$. Let $u_{0}$ and $H^{-}$be as in Proposition 3.1. Then for every $\Phi \in$ $\mathrm{C}_{0}^{\infty}\left(\mathbb{R}^{d}, \mathbb{R}^{d}\right)$,

$$
\int_{\mathbb{R}^{d}}\left\langle D \Phi \nabla u_{0} \cdot \nabla u_{0}\right\rangle-\frac{1}{2} \int_{\mathbb{R}^{d}} \operatorname{div} \Phi\left|\nabla u_{0}\right|^{2}=\lambda \int_{H^{-}} \operatorname{div} \Phi .
$$

Proof. Let $\Phi \in \mathrm{C}_{0}^{\infty}\left(\mathbb{R}^{d}, \mathbb{R}^{d}\right)$ and $P h i_{m}(x)=\Phi\left(\left(x-x_{m}\right) / r_{m}\right)$. For $m$ large enough, $\Phi_{m} \in \mathrm{C}_{0}^{\infty}\left(D_{1}, \mathbb{R}^{d}\right)$. Writing Euler-Lagrange's equation of $u$ with $\Phi_{m}$, we get

$$
\begin{gathered}
\int_{\mathbb{R}^{d}}\left\langle D \Phi_{m}(x) \nabla u(x) \cdot \nabla u(x) \mathrm{d} x\right\rangle-\frac{1}{2} \int_{\mathbb{R}^{d}} \operatorname{div} \Phi_{m}(x)|\nabla u(x)|^{2} \mathrm{~d} x \\
=\int_{\mathbb{R}^{d}} f(x)\left\langle\nabla u(x) \cdot \Phi_{m}(x)\right\rangle \mathrm{d} x+\lambda \int_{\Omega_{u}} \operatorname{div} \Phi_{m}(x) \mathrm{d} x .
\end{gathered}
$$

Setting $x=x_{m}+r_{m} y$ in these integrals, we obtain

$$
\begin{gathered}
\int_{\mathbb{R}^{d}}\left\langle D \Phi(y) \nabla u_{m} \cdot \nabla u_{m}\right\rangle-\frac{1}{2} \int_{\mathbb{R}^{d}} \operatorname{div} \Phi\left|\nabla u_{m}\right|^{2} \mathrm{~d} y \\
=r_{m} \int_{\mathbb{R}^{d}} f\left(x_{m}+r_{m} y\right)\left\langle\nabla u_{m}(y) \cdot \Phi(y)\right\rangle \mathrm{d} y+\lambda \int_{\left\{y, x_{m}+r_{m} y \in \Omega_{u}\right\}} \operatorname{div} \Phi(y) \mathrm{d} y .
\end{gathered}
$$

The last term converges to $\lambda \int_{H^{-}} \operatorname{div} \Phi$. The third term converges to 0 because $f$ and $\Phi$ are bounded and $\left\|\nabla u_{m}\right\|_{\infty}$ is uniformally bounded. Finally for the two first terms we use the convergence of $\nabla u_{m}$ to $\nabla u_{0}$. The result follows.

Proposition 3.4. Let $u_{0}$ and $H^{-}$be as in Proposition 3.1 and let $B\left(x_{1}, R\right) \subset H^{-}$almost-everywhere, then $u_{0}$ is harmonic on $B\left(x_{1}, R\right)$.

Proof. Let $v_{m}$ be defined by:

$$
v_{m}=u_{m} \text { on } \partial B\left(x_{1}, R\right), \Delta v_{m}=0 \text { in } B\left(x_{1}, R\right)
$$

Then, we have:

$$
\int_{B\left(x_{1}, R\right)}\left|\nabla v_{m}\right|^{2} \leq \int_{B\left(x_{1}, R\right)}\left|\nabla u_{m}\right|^{2} \leq C,\left\|v_{m}\right\|_{\infty} \leq\left\|u_{m}\right\|_{\infty} \leq C .
$$


It follows that, up to a subsequence, $v_{m}$ converges weakly in $\mathrm{H}^{1}\left(B\left(x_{1}, R\right)\right)$ to some $v_{0}$ which is harmonic and satisfies. $v_{0}=u_{0}$ on $\partial B\left(x_{1}, R\right)$. Since $v_{m}-v_{0}$ is harmonic in $B\left(x_{1}, R\right)$ and is equal to $u_{m}-u_{0}$ on $\partial B\left(x_{1}, R\right)$, we get:

$$
\int_{B\left(x_{1}, R\right)}\left|\nabla v_{m}-\nabla v_{0}\right|^{2} \leq \int_{B\left(x_{1}, R\right)}\left|\nabla u_{m}-\nabla u_{0}\right|^{2},
$$

which goes to 0 , thanks to the convergence in $L^{2}$ of $\nabla u_{m}$ (see Prop. 3.2).

Let, for $x \in \overline{B\left(x_{m}+r_{m} x_{1}, r_{m} R\right)}, w_{m}(x)=r_{m} v_{m}\left(\left(x-x_{m}\right) / r_{m}\right)$, for $x \in \partial B\left(x_{m}+r_{m} x_{1}, r_{m} R\right)$ we have, $w_{m}(x)=u(x)$. Therefore we can extend $w_{m}$ by $u$ outside $B\left(x_{m}+r_{m} x_{1}, r_{m} R\right)$. By minimality of $u$, we get,

$$
\int_{B\left(x_{m}+r_{m} x_{1}, r_{m} R\right)}\left(\frac{1}{2}|\nabla u|^{2}-f u\right) \leq \int_{B\left(x_{m}+r_{m} x_{1}, r_{m} R\right)}\left(\frac{1}{2}\left|\nabla w_{m}\right|^{2}-f w_{m}\right)+\lambda^{*}\left|B\left(x_{m}+r_{m} x_{1}, r_{m} R\right) \cap\{u=0\}\right| .
$$

By change of variables $x=x_{m}+r_{m}(x+y)$ in the integrals, we obtain:

$$
\int_{B\left(x_{1}, R\right)}\left(\frac{1}{2} r_{m}^{d}\left|\nabla u_{m}\right|^{2}-r_{m}^{d+1} f u_{m}\right) \leq \int_{B\left(x_{1}, R\right)}\left(\frac{1}{2} r_{m}^{d}\left|\nabla v_{m}\right|^{2}-r_{m}^{d+1} f v_{m}\right)+\lambda^{*} r_{m}^{d}\left|B\left(x_{1}, R\right) \backslash \Omega_{m}\right| .
$$

We divide by $r_{m}^{d}$ and let $m$ go to infinity. Since $B\left(x_{1}, R\right) \subset H^{-}$, and $\chi_{H^{-}}=\lim \chi_{\Omega_{m}}$ we deduce:

$$
\int_{B\left(x_{1}, R\right)}\left|\nabla u_{0}\right|^{2} \leq \int_{B\left(x_{1}, R\right)}\left|\nabla v_{0}\right|^{2},
$$

and we get that $u_{0}$ is harmonic in $B\left(x_{1}, R\right)$.

For the next proposition, we will suppose that $u \geq 0$ around the point where we study the blow-up.

Proposition 3.5. Let $u$ be a solution of $(\mathcal{P})$ and $D_{1}$ an open subset of $D$ such that $u$ is nonnegative in $D_{1}$. Let $x_{0} \in \partial^{*} \Omega_{u} \cap D_{1}$ and $\nu$ the outward unit normal to $\partial \Omega_{u}$ at $x_{0}$. Let $u_{0}$ the limit of a subsequence of $u_{m}$ for the blow-up relatively to $B\left(x_{0}, r_{m}\right)$. Then we have:

$$
u_{0}(x)=\left\{\begin{array}{cc}
-\sqrt{2 \lambda}(x, \nu), & x \in H^{-}=\left\{x \in \mathbb{R}^{d},(x, \nu)<0\right\} \\
0, & x \notin H^{-}
\end{array}\right.
$$

Proof. By Theorem $1.8 u$ is locally Lipschitz continuous in $D_{1}$.

Since $x_{0} \in \partial^{*} \Omega_{u}$, due to the properties on the reduced boundary, we have that, $\Omega_{m}=\left\{x, x_{0}+r_{m} x \in \Omega_{u}\right\}$ converges to $H^{-}=\{x ;(x . \nu)<0\}$ in $L_{\text {loc }}^{1}\left(\mathbb{R}^{d}\right)$ and almost everywhere (up to a sub-sequence). Up to a rotation of coordinates we can suppose that $\nu=-e_{1}=(-1,0, . ., 0)$ and so we have $H^{-}=\left\{x=\left(x_{1}, \ldots, x_{d}\right), x_{1}>0\right\}$. For almost every $x \notin H^{-}, u_{m}(x)=0$ when $m$ is large enough and so $u_{0}(x)=0$, by continuity we have $u_{0}(x)=0$ for all $x \notin H^{-}$.

Let $L=\left\{x \in \mathbb{R}^{d}, x_{1}=0\right\}$. At this point, $u_{0}$ is harmonic and nonnegative on $\left[x_{1}>0\right]$, vanishes on $\left\{x \in \mathbb{R}^{d}, x_{1} \leq 0\right\}$ and is globally Lipschitz. By a classical reflexion argument and Liouville's Theorem, we get that there exists $a_{1} \geq 0$ such that $u_{0}(x)=a_{1} x_{1}$. Let $\varphi \in \mathrm{C}_{0}^{\infty}\left(\mathbb{R}^{d}, R\right)$ and $\Phi=-\varphi e_{1}$. We write the Euler's equation for $u_{0}(20)$ with this $\Phi$ :

$$
\begin{gathered}
\int_{H^{-}}\left(D \Phi \nabla u_{0} \cdot \nabla u_{0}\right)=\int_{L} a_{1}^{2} \varphi \\
\int_{H^{-}} \operatorname{div} \Phi\left|\nabla u_{0}\right|^{2}=\int_{L} a_{1}^{2} \varphi, \lambda \int_{H^{-}} \operatorname{div} \Phi=\lambda \int_{L} \varphi .
\end{gathered}
$$

We deduce that $a_{1}^{2}=2 \lambda$ and because $u_{0} \geq 0$ on $D_{1}$ we get (21). 


\section{Pseudo-Penalized problems}

We will now show that a solution of $(\mathcal{P})$ is also a solution of a "pseudo-penalized" problem. In this section $D_{1}$ will be such that:

$$
\left.\begin{array}{c}
D_{1} \subset D \text { open and bounded with } \partial D_{1} \text { regular } \\
u \text { not identically equal to } 0 \text { on } \partial D_{1} \\
\exists h_{0} \text { such that } 0<h_{0}<\left|D_{1} \cap \Omega_{u}\right|<\left|D_{1}\right|-h_{0} \\
0<h_{0}<\left|\left(D \backslash D_{1}\right) \cap \Omega_{u}\right|<\left|D \backslash D_{1}\right|-h_{0}
\end{array}\right\} \text {. }
$$

Remark 4.1. These technical conditions just mean that there is a non-negligible part of $D_{1}$ intersecting $\Omega_{u}$ and a non negligible part intersecting the set $\{u=0\}$. It is, in particular, satisfied for all balls of small radius centered on $\partial^{*} \Omega_{u}$.

First, we give a technical lemma that we will use repeatedly in the next proofs. It is related to the Euler-Lagrange's equation (8), except that here we only assumed it to be satisfied in $D_{1}$. The proof is the same as the one of (6) and we do not reproduce it here.

Lemma 4.2. Let $v \in \mathrm{H}_{0}^{1}(D)$ be such that there exists a $\lambda_{v} \geq 0$ such that, for all $\Phi \in \mathrm{C}_{0}^{\infty}\left(D_{1}, \mathbb{R}^{d}\right)$,

$$
\int_{D_{1}}(D \Phi \nabla v \cdot \nabla v)-\frac{1}{2} \int_{D_{1}}|\nabla v|^{2} \operatorname{div} \Phi=\int_{D_{1}} f \nabla v \cdot \Phi+\lambda_{v} \int_{\Omega_{v}} \operatorname{div} \Phi .
$$

Let $v_{t}(x)=v(x+t \Phi(x))$ for $\Phi \in \mathrm{C}_{0}^{\infty}\left(D_{1}, \mathbb{R}^{d}\right)$. Then

$$
\begin{aligned}
J\left(v_{t}\right) & =\int_{D_{1}}(D \Phi \nabla v \cdot \nabla v)-\frac{1}{2} \int_{D_{1}}|\nabla v|^{2} \operatorname{div} \Phi-\int_{D_{1}} f \nabla v \cdot \Phi+o(t) \\
& =J(v)+t \lambda_{v} \int_{\Omega_{v}} \operatorname{div} \Phi+o(t), \\
\left|\Omega_{v_{t}}\right| & =|\Omega|-t \int_{\Omega_{v}} \operatorname{div} \Phi+o(t) .
\end{aligned}
$$

We will now see that if $u$ is a solution of $(\mathcal{P})$, it is also a solution of a pseudo-penalized problem. More precisely, we know that $J(u)-J(v) \leq 0$ if $\left|\Omega_{v}\right|<a$. We will see that we can control $J(u)-J(v)$ in term of $\left|\Omega_{v}\right|-\left|\Omega_{u}\right|$.

We will suppose from now and to the end of this section, except for the last Remark 4.6, that $u$ is a solution of $(\mathcal{P})$ with $\lambda>0$ and $D_{1}$ verifies condition $(22)$. We define:

$$
\mathcal{F}_{0}=\left\{v \in \mathrm{H}_{0}^{1}(D) ; u-v \in \mathrm{H}_{0}^{1}\left(D_{1}\right)\right\},
$$

and, for $h<h_{0}$,

$$
\begin{gathered}
\mu(h)=\sup \left\{\mu \geq 0 ; J(u)+\mu\left|\Omega_{u}\right| \leq J(v)+\mu\left|\Omega_{v}\right|, \forall v \in \mathcal{F}_{0}, a-h \leq\left|\Omega_{v}\right| \leq a\right\}, \\
\lambda^{*}(h)=\inf \left\{\lambda^{*} \geq 0 ; J(u) \leq J(v)+\lambda^{*}\left(\left|\Omega_{v}\right|-a\right)^{+}, \forall v \in \mathcal{F}_{0},\left|\Omega_{v}\right| \leq a+h\right\} .
\end{gathered}
$$

So by definition we have: for every $v \in \mathcal{F}_{0}$

$$
\begin{gathered}
a-h \leq\left|\Omega_{v}\right| \leq a \Longrightarrow J(u)+\mu(h)\left|\Omega_{u}\right| \leq J(v)+\mu(h)\left|\Omega_{v}\right| \\
a \leq\left|\Omega_{v}\right| \leq\left|\Omega_{u}\right|+a \Longrightarrow J(u)+\lambda^{*}(h)\left|\Omega_{u}\right| \leq J(v)+\lambda^{*}(h)\left|\Omega_{v}\right| .
\end{gathered}
$$


The main result on $\mu(h)$ and $\lambda(h)$ is the following theorem:

Proposition 4.3. The function $\mu$ is nondecreasing function, $\lambda^{*}$ is non-increasing and for some $h_{0}$ and $\left.h \in\right] 0, h_{0}[$,

$$
0<\mu(h) \leq \lambda \leq \lambda^{*}(h)<+\infty .
$$

Moreover $\lim _{h \rightarrow 0} \mu(h)=\lim _{h \rightarrow 0} \lambda^{*}(h)=\lambda$.

We start by proving that the sets of $\lambda^{*}$ appearing in the definition of $\lambda^{*}(h)$ is not empty. This is done also in [13] for $D_{1}=D$. Here the proof is slightly different and is more local. Most of the arguments will be used later for the study of $\lambda^{*}(h)$.

Proposition 4.4. Let $u$ be a solution of $(\mathcal{P})$. There exists $\lambda^{*}>0$ such that

$$
J(u)+\lambda^{*}\left|\Omega_{u}\right| \leq J(v)+\lambda^{*}\left|\Omega_{v}\right|,
$$

for every $v \in \mathcal{F}_{0}$ with $\left|\Omega_{v}\right| \geq a$. Moreover we have $\lambda \leq \lambda^{*}$.

Proof. Let $\lambda_{n}^{*}$ be an increasing sequence and $\lim _{n \rightarrow \infty} \lambda_{n}^{*}=+\infty$. Let $v_{n} \in \mathcal{F}_{0}$ such that

$$
J\left(v_{n}\right)+\lambda_{n}^{*}\left(\left|\Omega_{v_{n}}\right|-a\right)^{+} \leq J(v)+\lambda_{n}^{*}\left(\left|\Omega_{v}\right|-a\right)^{+},
$$

for all $v \in \mathcal{F}_{0}$ (existence of $v_{n}$ is straightforward since $\mathcal{F}_{0}$ is closed). If there exists $n \geq 0$ such that $\left|\Omega_{v_{n}}\right| \leq a$, we have

$$
J(u) \leq J\left(v_{n}\right) \leq J(v)+\lambda_{n}^{*}\left(\left|\Omega_{v}\right|-a\right)^{+},
$$

for all $v \in \mathcal{F}_{0}$ and we get (26) with $\lambda^{*}=\lambda_{n}^{*}$. So we argue by contradiction and we suppose that, for all $n \geq 0$, $\left|\Omega_{v_{n}}\right|>a$. Let $w \in \mathcal{F}_{0}$ such that $-\Delta w=f$ in $D_{1}$. We have:

$$
0 \leq \lambda_{n}^{*}\left(\left|\Omega_{v_{n}}\right|-a\right) \leq J(u)-J\left(v_{n}\right) \leq J(u)-J(w),
$$

so we can deduce that $\lim \left|\Omega_{v_{n}}\right|=a$. Since $J\left(v_{n}\right) \leq J(u)$ we have, up to a sub-sequence, that $v_{n}$ converges weakly in $\mathrm{H}^{1}\left(D_{1}\right)$ and a.e to $v \in \mathcal{F}_{0}$. Then $\left|\Omega_{v}\right| \leq a$ and,

$$
J(v) \leq \liminf _{n \rightarrow \infty} J\left(v_{n}\right) \leq J(u),
$$

so $v$ is also a solution of $(\mathcal{P})$ and $\left|\Omega_{v}\right|=a$. According to Proposition 1.2 there exists a $\lambda_{v}$ such that $v$ satisfies Euler-Lagrange's equation in $D$. Because of the condition on $\left|\left(D \backslash D_{1}\right) \cap \Omega_{u}\right|=\left|\left(D \backslash D_{1}\right) \cap \Omega_{v}\right|>0$ there exists $\varphi \in \mathrm{C}_{0}^{\infty}\left(D \backslash \overline{D_{1}}, \mathbb{R}^{d}\right)$ such that $\int_{\Omega_{u}} \operatorname{div} \varphi \neq 0$. Because $u=v$ outside $D_{1}$, we get, writing (8) with $\varphi$ for $u$ and $v$, that $\lambda_{v}=\lambda$. Moreover, since $J\left(v_{n}\right) \leq J(w)$ for $w \in \mathcal{F}_{0}$ with $\left|\Omega_{w}\right| \leq\left|\Omega_{v_{n}}\right|$, as in Proposition 1.2, one can prove that $v_{n}$ satisfies Euler-Lagrange's equation (23) with some $\lambda_{v_{n}}=\lambda_{n}$. Let us show that $\lambda_{n} \geq \lambda_{n}^{*}$. Let $\varphi \in \mathrm{C}_{0}^{\infty}\left(D_{1}\right)$ such that $\int_{\Omega_{v_{n}}} \operatorname{div} \varphi>0$ ( $\varphi$ exists for $n$ large enough, thanks to $\left|D_{1} \cap \Omega_{u}\right|<\left|D_{1}\right|$ and $\left.\lim \left|\Omega_{v_{n}}\right|=\left|\Omega_{u}\right|\right)$. Set $v_{n}^{t}=v_{n}(x+t \varphi(x))$. According to Lemma 4.2 and because $\left|\Omega_{v_{n}}\right|>a$, we have for $t>0$ small enough

$$
\left|\Omega_{v_{n}^{t}}\right|=\left|\Omega_{v_{n}}\right|-t \int_{\Omega_{v_{n}}} \operatorname{div} \varphi+o(t)>a .
$$

Using the definition of $v_{n}$ with $v=v_{n}^{t}$ and Lemma 4.2, we get:

$$
J\left(v_{n}\right) \leq J\left(v_{n}\right)+t \lambda_{n} \int_{\Omega_{v_{n}}} \operatorname{div} \varphi-\lambda_{n}^{*} t \int_{\Omega_{v_{n}}} \operatorname{div} \varphi+o(t),
$$

and so $\lambda_{n} \geq \lambda_{n}^{*}$. 
We now want to show that $\lim \lambda_{n}=\lambda$. Then using $\lambda_{n} \geq \lambda_{n}^{*}$ and $\lim \lambda_{n}^{*}=+\infty$, we will get a contradiction. For this we just have to show that $v_{n}$ converge in the norm of $\mathrm{H}_{0}^{1}(D)$. Indeed let $\Phi \in \mathrm{C}_{0}^{\infty}\left(D_{1}, \mathbb{R}^{d}\right)$ such that $\int_{\Omega_{v}} \operatorname{div} \Phi>0$. Writing Euler's equation (23) for $v_{n}$, we get:

$$
\int_{D_{1}}\left(D \Phi \nabla v_{n} \cdot \nabla v_{n}\right)-\frac{1}{2} \int_{D_{1}}\left|\nabla v_{n}\right|^{2} \operatorname{div} \Phi-\int_{D_{1}} f \nabla v_{n} \cdot \Phi=\lambda_{n} \int_{\Omega_{v_{n}}} \operatorname{div} \Phi,
$$

and, letting $n$ goes to infinity we get:

$$
\left(\lim _{n \rightarrow \infty} \lambda_{n}\right) \int_{D_{1}} \operatorname{div} \Phi=\int_{D_{1}}(D \Phi \nabla v \cdot \nabla v)-\frac{1}{2} \int_{D_{1}}|\nabla v|^{2} \operatorname{div} \Phi-\int_{D_{1}} f \nabla v \cdot \Phi,
$$

and so, using Euler's equation for $v$ in $D, \lim _{n \rightarrow \infty} \lambda_{n}=\lambda$.

To show the strong convergence of $v_{n}$, because of the weak convergence, we just have to show the convergence of the norm of $\nabla v_{n}$. For this we just write: $J\left(v_{n}\right) \leq J(v)$, so

$$
\frac{1}{2} \int_{D}\left|\nabla v_{n}\right|^{2} \leq \frac{1}{2} \int_{D}|\nabla v|^{2}+\int_{D} f\left(v-v_{n}\right)
$$

and the last term goes to 0 (by weak convergence of $v_{n}$ ).

We now prove that $\lambda^{*} \geq \lambda$. For this let, $\varphi \in \mathrm{C}_{0}^{\infty}\left(D_{1}, \mathbb{R}^{d}\right)$ such that $\int_{\Omega_{u}} \operatorname{div} \varphi<0$ and $u_{t}(x)=u(x+t \varphi(x))$. Using Lemma 4.2, for $t$ small enough,

$$
\left|\Omega_{u_{t}}\right|=\left|\Omega_{u}\right|-t \int_{\Omega_{u}} \operatorname{div} \varphi+o(t) \geq\left|\Omega_{u}\right| .
$$

Writing

$$
J(u) \leq J\left(u_{t}\right)+\lambda^{*}\left(\left|\Omega_{u_{t}}\right|-a\right),
$$

and using Lemma 4.2 we get:

$$
0 \leq t\left(\lambda-\lambda^{*}\right) \int_{\Omega_{u}} \operatorname{div} \varphi+o(t)
$$

and so $\lambda \leq \lambda^{*}$.

Proof of Proposition 4.3. We showed, in the previous proposition, that, for $h>0, \lambda \leq \lambda^{*}(h)<+\infty$. The fact that $\lambda^{*}$ is non-increasing and $\mu$ is nondecreasing comes directly from the definition. We will now show that $\mu(h) \leq \lambda$. Let $\varphi \in \mathrm{C}_{0}^{\infty}\left(D_{1}\right)$ such that $\int_{\Omega_{u}} \operatorname{div} \varphi>0$ and $u_{t}(x)=u(x+t \varphi(x))$. From Lemma 4.2 we have for $t$ small enough:

$$
\left|\Omega_{u}\right|-h \leq\left|\Omega_{u_{t}}\right|=\left|\Omega_{u}\right|-t \int_{\Omega_{u}} \operatorname{div} \varphi+o(t) \leq\left|\Omega_{u}\right|
$$

Writing

$$
J(u)+\mu(h)\left|\Omega_{u}\right| \leq J\left(u_{t}\right)+\mu(h)\left|\Omega_{u_{t}}\right|
$$

and using Lemma 4.2, we get:

$$
0 \leq t(\lambda-\mu(h)) \int_{\Omega_{u}} \varphi+o(t),
$$

and so $\mu(h) \leq \lambda$. We will study the limit of $\lambda^{*}(h)$ and $\mu(h)$ as $h$ tends to 0 , and this will give us directly $\mu(h)>0$ for $h$ small enough. We will begin with the limit of $\lambda^{*}(h)$. The proof is very close to the one of Proposition 4.4 . Let $h_{n}$ decrease to 0 . Since $\lambda^{*}$ is non-increasing and $\lambda \leq \lambda^{*}(h)$ we just have to show that $\lim \lambda^{*}\left(h_{n}\right) \leq \lambda$ for a sub-sequence of $h_{n}$. 
Let $\varepsilon \in] 0, \lambda\left[\right.$. By minimization, one proves existence of $v_{n} \in \mathcal{F}_{0}$, with $\left|\Omega_{v_{n}}\right| \leq a+h_{n}$ such that:

$$
J\left(v_{n}\right)+\left(\lambda^{*}\left(h_{n}\right)-\varepsilon\right)\left(\left|\Omega_{v_{n}}\right|-a\right)^{+} \leq J(v)+\left(\lambda^{*}\left(h_{n}\right)-\varepsilon\right)\left(\left|\Omega_{v}\right|-a\right)^{+},
$$

for all $v \in \mathcal{F}_{0}$ such that $\left|\Omega_{v}\right| \leq a+h_{n}$.

First we have that $\left|\Omega_{v_{n}}\right|>a$. If $\left|\Omega_{v_{n}}\right| \leq a$, we would have, for all $v \in \mathcal{F}_{0}$ with $\left|\Omega_{v}\right| \leq a+h_{n}$,

$$
J(u) \leq J\left(v_{n}\right)=J\left(v_{n}\right)+\left(\lambda^{*}\left(h_{n}\right)-\varepsilon\right)\left(\left|\Omega_{v}\right|-a\right)^{+} \leq J(v)+\left(\lambda^{*}\left(h_{n}\right)-\varepsilon\right)\left(\left|\Omega_{v_{n}}\right|-a\right)^{+},
$$

which contradicts the definition of $\lambda^{*}\left(h_{n}\right)$.

We have:

$$
J\left(v_{n}\right)+\left(\lambda^{*}\left(h_{n}\right)-\varepsilon\right)\left(\left|\Omega_{v_{n}}\right|-a\right) \leq J(u)
$$

Up to a sub-sequence $v_{n}$ converge weakly in $\mathrm{H}^{1}\left(D_{1}\right)$ to a $v$, moreover we have that $v \in \mathcal{F}_{0},\left|\Omega_{v}\right| \leq \lim \left|\Omega_{v_{n}}\right|=a$, $J(v) \leq \liminf J\left(v_{n}\right)$ and, passing to the limit in $(27)$, we obtain

$$
J(v) \leq J(u)=\min \left\{J(w), w \in \mathrm{H}_{0}^{1}(D),\left|\Omega_{w}\right| \leq a\right\} \leq J(v)
$$

and we have $\left|\Omega_{v}\right|=a$, since from $\left|\Omega_{v}\right|<a$, we would easily prove that $-\Delta v=f$ in $D$ and contradict assumption (7). Like in Proposition 4.4 we write Euler's equation in $D$ for $v$ with a $\lambda_{v}$ and we get that $\lambda=\lambda_{v}$.

We can write an Euler's equation in $D_{1}$ for $v_{n}$ with some $\lambda_{n}$ : if $w \in \mathcal{F}_{0}$ such that $\left|\Omega_{w}\right| \leq\left|\Omega_{v}\right|$ we have $J\left(v_{n}\right) \leq J(w)$. Also $v_{n}$ converges strongly in $\mathrm{H}_{0}^{1}(D)$ since, as in Proposition $4.4, J\left(v_{n}\right) \leq J(v)$. So, writing the Euler's equation and letting $n$ go to infinity, we get that $\lim \lambda_{n}=\lambda$. To conclude, we just have to see that $\lambda_{n} \geq\left(\lambda^{*}\left(h_{n}\right)-\varepsilon\right)$. Let $\varphi \in \mathrm{C}_{0}^{\infty}\left(D_{1}\right)$ such that $\int_{\Omega_{v_{n}}} \operatorname{div} \varphi>0\left(\varphi\right.$ exists thanks to the hypothesis on $\left.\left|D_{1} \cap \Omega_{u}\right|\right)$ and $v_{n}^{t}=v_{n}(x+t \varphi(x))$. According to Lemma 4.2 and because $\left|\Omega_{v_{n}}\right|>a$, we have for $t>0$ small enough

$$
\left|\Omega_{v_{n}^{t}}\right|=\left|\Omega_{v_{n}}\right|-t \int_{\Omega_{v_{n}}} \operatorname{div} \varphi+o(t)>a .
$$

Using the minimality of $v_{n}$ with respect to $v_{n}^{t}$ Lemma 4.2 , we get:

$$
\left.J\left(v_{n}\right) \leq J\left(v_{n}\right)+t \lambda_{n} \int_{\Omega_{v_{n}}} \operatorname{div} \varphi+o(t)-\left(\lambda^{*}\left(h_{n}\right)-\varepsilon\right) t \int_{\Omega_{v_{n}}} \operatorname{div} \varphi+o(t)\right),
$$

and so $\lambda_{n} \geq\left(\lambda\left(h_{n}\right)-\varepsilon\right)$.

Now we study the limit of $\mu(h)$. Many arguments are similar to those used in the proof of Proposition 4.4 and in the study of $\lambda^{*}(h)$. Let $h_{n}$ decrease to 0 and $\varepsilon>0$. Let $v_{n} \in \mathcal{F}_{0}$, with $\left|\Omega_{v_{n}}\right| \leq a$, solution of the following minimization problem:

$$
J\left(v_{n}\right)+\left(\mu\left(h_{n}\right)+\varepsilon\right)\left(\left|\Omega_{v_{n}}\right|-\left(a-h_{n}\right)\right)^{+} \leq J(v)+\left(\mu\left(h_{n}\right)+\varepsilon\right)\left(\left|\Omega_{v}\right|-\left(a-h_{n}\right)\right)^{+},
$$

for all $v \in \mathcal{F}_{0},\left|\Omega_{v}\right| \leq a$. First we have $\left|\Omega_{v_{n}}\right|<a$ : indeed, if $\left|\Omega_{v_{n}}\right|=a$, we get, taking $v \in \mathcal{F}_{0}$ with $a-h_{n} \leq$ $\left|\Omega_{v}\right| \leq a$,

$$
\begin{aligned}
J(u) & \leq J\left(v_{n}\right) \\
& \leq J(v)+\left(\mu\left(h_{n}\right)+\varepsilon\right)\left(\left|\Omega_{v}\right|-\left(a-h_{n}\right)\right)^{+}-\left(\mu\left(h_{n}\right)+\varepsilon\right)\left(\left|\Omega_{v_{n}}\right|-\left(a-h_{n}\right)\right)^{+} \\
& =J(v)+\left(\mu\left(h_{n}\right)+\varepsilon\right)\left(\left|\Omega_{v}\right|-\left|\Omega_{u}\right|\right),
\end{aligned}
$$

which contradicts the definition of $\mu\left(h_{n}\right)$. 
We now see that: $\left|\Omega_{v_{n}}\right| \geq a-h_{n}$. By contradiction if $\left|\Omega_{v_{n}}\right|<a-h_{n}$, we would have for all $\varphi \in \mathrm{C}_{0}^{\infty}\left(D_{1}\right)$, with $0<|\{\varphi \neq 0\}| \leq\left(a-h_{n}\right)-\left|\Omega_{v_{n}}\right|, v_{n}+t \varphi \in \mathcal{F}_{0},\left|\Omega_{v_{n}+t \varphi}\right| \leq a-h_{n}$ and, by minimality of $v_{n}, J\left(v_{n}\right) \leq$ $J\left(v_{n}+t \varphi\right), t \in \mathbb{R}$. So we deduce that

$$
-\Delta v_{n}=f \text { in } D_{1}
$$

and because $J(u) \leq J\left(v_{n}\right)$, by uniqueness of the solution of the minimum of $J$ in $\mathcal{F}_{0}$ we get also that $u=v$ and

$$
-\Delta u=f \text { in } D_{1}
$$

From this we can deduce that $u$ is locally Lipschitz in $D_{1}$ and, by Theorem $2.4, \Omega_{u}$ has finite perimeter in $D_{1}$. In particular, $\partial^{*} \Omega_{u} \cap D_{1}$ is not empty. Let $x_{0} \in \partial^{*} \Omega_{u} \cap D_{1}$ and let $r_{m}$ go to 0 . We study the blow up of $u$ relatively to $B\left(x_{0}, r_{m}\right)$ and denote by $u_{0}$ a limit (up to a sub-sequence). Since

$$
-\Delta u_{m}(x)=r_{m} f\left(x_{0}+r_{m} x\right), \text { in } B(0, R)
$$

as soon as $B\left(x_{0}, r_{m} R\right) \subset D_{1}$, we get that $u_{0}$ is harmonic in $\mathbb{R}^{d}$. If we write the Euler's equation for $u_{0}$ we get for $\Phi \in \mathrm{C}_{0}^{\infty}\left(\mathbb{R}^{d}, \mathbb{R}^{d}\right)$ :

$$
\int_{\mathbb{R}^{d}}\left(D \Phi \nabla u_{0}, \nabla u_{0}\right)-\frac{1}{2} \int_{\mathbb{R}^{d}} \operatorname{div} \Phi\left|\nabla u_{0}\right|^{2}=\lambda \int_{H^{-}} \operatorname{div} \Phi
$$

Using that $u_{0}$ is harmonic (and so $u_{0}$ is $\mathrm{C}^{\infty}$ ) we get that the left hand side is equal to 0 , which is a contradiction because $\lambda>0$ and $H^{-}$is a half-space. (Note that if we know that $u \geq 0$ we can use Prop. 3.5.)

Now we known that $a-h_{n} \leq\left|\Omega_{v_{n}}\right|<a$ and we may use it in (28). For instance, we have $J\left(v_{n}\right) \leq J(v)$ for all $v \in \mathcal{F}_{0}$ with $\left|\Omega_{v}\right| \leq\left|\Omega_{v_{n}}\right|$. Therefore there exists $\lambda_{n}$ such that $v_{n}$ satisfies the Euler's equation in $D_{1}$. We take $\varphi \in \mathrm{C}_{0}^{\infty}\left(D_{1}, \mathbb{R}^{d}\right)$ with $\int_{\Omega_{v_{n}}} \operatorname{div} \varphi<0$ and $v_{n}^{t}(x)=v_{n}(x+t \varphi(x)), x \in D_{1}$. Since $\Omega_{v_{n}}<a$, by Lemma 4.2 , we have $\left|\Omega_{v_{n}^{t}}\right| \leq a$ for t small enough and, (as in the study of $\lambda^{*}$ )

$$
\lambda_{n} \leq \mu\left(h_{n}\right)+\varepsilon
$$

As before $v_{n}$ weakly converges (up to a sub-sequence) to some $v \in \mathcal{F}_{0}$ with $\left|\Omega_{v}\right|=a$ and

$$
J(v) \leq \liminf _{n \rightarrow \infty} J\left(v_{n}\right) \leq J(u)
$$

(because $\lim \left|\Omega_{v_{n}}\right|=a$ ). Again, we get that $v$ satisfies Euler's equation with the same $\lambda$ as $u$. We have, as before, convergence in the norm of $\mathrm{H}_{0}^{1}(D)$ by using

$$
J\left(v_{n}\right) \leq J(v)+\left(\mu\left(h_{n}\right)-\left(a-h_{n}\right)\right)\left(\left|\Omega_{v_{n}}\right|-a\right),
$$

and we conclude in the same way $\lim \lambda_{n}=\lambda$. This, together with $(29)$ and $\lambda>0$ implies that $\mu(h)>0$ for $h$ small enough.

Remark 4.5. We can show, using the same methods, that in fact we have $\mu(h)>0$ for every $h>0$.

Remark 4.6. In the proof of Proposition 4.3, we use $\lambda>0$ only for $\mu(h)$. So, if $u$ is a solution of $(\mathcal{P})$ with $\lambda=0$, we get the existence of $\lambda^{*}(h)$ such that $(25)$ holds and $\lim _{h \rightarrow 0} \lambda^{*}(h)=0$. 


\section{REgularity OF THE BOUNDARY}

In this section we study the regularity of the boundary of $\Omega_{u}$ with $u$ a solution of $(\mathcal{P})$ in regions where $u$ does not change its sign. The main result will be the following theorem:

Theorem 5.1. Let $u$ be a solution of $(\mathcal{P})$ with $\lambda>0$ and let $D_{1} \subset D$ satisfy conditions (22) and:

$$
D_{1} \subset\{u \geq 0\}
$$

Then: $\Omega_{u}$ has locally finite perimeter in $D_{1}$ and

$$
\begin{gathered}
\mathcal{H}^{d-1}\left(\left(\partial \Omega_{u} \backslash \partial^{*} \Omega_{u}\right) \cap D_{1}\right)=0, \\
\Delta u+f \chi_{\Omega_{u}}=\sqrt{2 \lambda} \mathcal{H}^{d-1}\left\lfloor\partial \Omega_{u}, \text { in } \mathcal{D}^{\prime}\left(D_{1}\right) .\right.
\end{gathered}
$$

The reduced boundary $\partial^{*} \Omega_{u}$ is a $C^{1, \alpha}$ hypersurface $(\alpha>0)$. If moreover $d=2$, we have $\partial^{*} \Omega_{u}=\partial \Omega_{u}$ and so $\partial \Omega_{u}$ is regular in $D_{1}$.

Remark 5.2. If we know that $u \geq 0$ on $D$, Theorem 5.1 is true with $D$ instead of $D_{1}$ (we apply the theorem with $D_{1}$ equal to balls with center on $\partial \Omega_{u}$ ).

The proofs of the following propositions and lemmas are very close to the ones in $[2,10]$. The main differences is that we have "pseudo-penalization" conditions (24) and (25). Here $\mu(h) \neq \lambda^{*}(h)$ in general, while equality occurs in $[2,10]$ (in fact with a term like $\int_{\Omega_{u}} g^{2}$ with $g^{2}>c>0$ ). So we have to verify that the approach in $[2,10]$ works even if $\lambda^{*}(h) \neq \mu(h)$. A main point here though is that $\mu(h)>0$. We begin with a technical proposition (as in [2]):

Proposition 5.3. Let $u$ and $D_{1}$ as in Theorem 5.1. Then for all $0<\tau<1$ there exist $C>0, r_{0}>0$ such that for all balls with $B\left(x_{1}, r\right) \subset D_{1}$ and $r \leq r_{0}$, we have:

$$
\frac{1}{r} f_{\partial B\left(x_{1}, r\right)} u \leq C,
$$

implies $u=0$ on $B\left(x_{1}, \tau r\right)$.

Remark 5.4. This result essentially means that the averages are bounded from below. This is one main step in proving that $\nabla u$ does not degenerate near the boundary.

Proof. The proof for $\tau=1 / 4$ is the same as in Lemma 2.8 of [10] with $g=\sqrt{2 \mu(h)}$, where $h=\omega_{d} r_{0}^{d}$, (by Prop. $4.3 \mu(h)>0$ if $r_{0}$ is small enough), and the same $f$. In this article the authors study $\left(B_{r}=B\left(x_{1}, r\right)\right)$ minimizers of:

$$
J_{r}(v)=\int_{B_{r / 2}}\left(|\nabla v|^{2}-2 f v+g^{2} \chi_{\Omega_{v}}\right), v \in \mathrm{H}^{1}\left(B_{r / 2}\right) .
$$

So we have, using Proposition 4.3, in our case that, for $r \leq r_{0}$ :

$$
J_{r}(u) \leq J_{r}(w)
$$

for all $w \in \mathcal{F}_{0}$ if $a-\omega_{d} r_{0}^{d} \leq\left|\Omega_{v}\right| \leq\left|\Omega_{u}\right|$. In [10] the authors use only the following perturbation: $w=\min \{u, v\}$ on $B_{r / 2}$ and $w=u$ outside $B_{r / 2}$ for a $v \geq 0$ such that $u<v$ on $\partial B_{r / 2}$. Using the same $w$, we have $w \in \mathcal{F}_{0}$ and $a-\omega_{d} r_{0}^{d} \leq\left|\Omega_{w}\right| \leq\left|\Omega_{u}\right|$ so, using (24) and the same proof we obtain the proposition. 
Proposition 5.5. There exist $C_{1}, C_{2}, r_{0}$ such that, for every $B\left(x_{0}, r\right) \subset D_{1}$ and $r \leq r_{0}$, we have

$$
0<C_{1} \leq \frac{\left|B\left(x_{0}, r\right) \cap \Omega_{u}\right|}{\left|B\left(x_{0}, r\right)\right|} \leq C_{2}<1 .
$$

Moreover we have:

$$
\mathcal{H}^{d-1}\left(\left(\partial \Omega_{u} \backslash \partial^{*} \Omega_{u}\right) \cap D_{1}\right)=0
$$

Proof. We know that $\Omega_{u}$ has finite perimeter (see Th. 2.4). The proof of the first part is the same as Lemma 3.7 in [2] (see also [10], Lem. 2.10). The second part comes from the theory of sets with finite perimeter (see 5.8 in $[6])$.

Theorem 5.6. We have in the sense of distribution in $D_{1}$ that:

$$
\Delta u+f \chi_{\Omega_{u}}=\sqrt{2 \lambda} \mathcal{H}^{d-1} \cdot\left\lfloor\partial \Omega_{u} .\right.
$$

Proof. The proof is the same as in Theorem 2.13 in [10] see also [2] (4.7,5.5). The steps are as follows: we show that the measure $\Delta u+\chi_{\Omega_{u}}$ is absolutely continuous with respect to $\mathcal{H}^{d-1} .\left\lfloor\partial \Omega_{u}\right.$ (both are radon measures). And, using Proposition 3.5, we get that the derivative of $\Delta u+\chi_{\Omega_{u}}$ is $\sqrt{2 \lambda}$ on $\partial^{*} \Omega_{u}$ and therefore almost everywhere.

At this stage, we showed that $u$ is what is called in [10] and in [2] a weak solution (see Def. 3.1 in [10] and 5.1 in [2]). We directly get the following regularity for $\partial D_{1}$.

Theorem 5.7. Let $u$ as in Theorem 5.1, then

1) $\partial^{*} \Omega_{u} \cap D_{1}$ is a $C^{1, \alpha}$ hypersurface for some $\alpha>0$ and $\mathcal{H}^{d-1}\left(\left(\partial \Omega_{u} \backslash \partial^{*} \Omega_{u}\right) \cap D_{1}\right)=0$;

2) If $d=2$ then $\partial \Omega_{u}=\partial^{*} \Omega_{u}$ and so $\partial \Omega_{u}$ is regular in $D_{1}$.

Remark 5.8. If $f$ is more regular around $\Omega_{u}$, then so is $\partial^{*} \Omega_{u}$ (or $\partial \Omega_{u}$ when $\left.d=2\right)$.

Proof. The first point directly comes from [2], 6-8, generalized in [10], Section 5. One important thing is that the regularity of $\partial^{*} \Omega_{u}$ is shown for "weak solution" in [2], and we have proved that we do have such weak solutions here.

For the second point, we have to generalize Theorem 6.6 in [2] and his Corollary 6.7. The corollary is deduced from the theorem exactly in the same way as in [2].

So, when $d=2$, we have to show that:

$$
\lim _{r \rightarrow 0} \int_{B_{r} \cap \Omega_{u}} \max \left\{\lambda-|\nabla u|^{2}, 0\right\}=0 .
$$

For $B_{r}=B\left(x_{0}, r\right)$ with $x_{0} \in \partial \Omega_{u}$. We take $\zeta \in C_{0}^{1}\left(D_{1}\right)$ as in [2] and the same $v=\max \{u-\varepsilon \zeta, 0\}$. Then we have $\left|\Omega_{u}\right|-\left|\Omega_{v}\right|=|\{0<u \leq \varepsilon \zeta\}| \leq|\{\zeta \neq 0\}|$, so using the definition of $\mu(h)$ with $h=|\{\zeta \neq 0\}|$ we get, as in $[2]$ :

Now, using $\lambda \geq \mu(h)$ :

$$
\int_{\{0<u \leq \varepsilon \zeta\}}\left(\mu(h)-\frac{|\nabla u|^{2}}{2}\right) \leq \frac{\varepsilon^{2}}{2} \int_{\{u>\varepsilon \zeta\}}|\nabla \zeta|^{2} .
$$

$$
\int_{\{0<u \leq \varepsilon \zeta\}}\left(\lambda-\frac{|\nabla u|^{2}}{2}\right) \leq \frac{\varepsilon^{2}}{2} \int_{\{u>\varepsilon \zeta\}}|\nabla \zeta|^{2}+(\lambda-\mu(h)) h .
$$

Exactly as in [2], we take for $r \leq \rho \leq R$,

$$
\zeta(x)=\left\{\begin{array}{ccc}
\frac{\log \left(\rho /\left|x-x_{0}\right|\right)}{\log (\rho / r)} & \text { for } & r<\left|x-x_{0}\right| \leq \rho \\
1 & \text { for } & \left|x-x_{0}\right| \leq r
\end{array}\right.
$$


We have $u(x) \leq C r$ in $B_{r}\left(x_{0} \in \partial \Omega_{u}\right.$ and $u$ is Lipschitz $)$ and we choose $\varepsilon=C r$. We get, with this choice of $\zeta$ and $\varepsilon$ :

$$
\begin{array}{r}
\int_{B_{r} \cap \Omega_{u}} \max \left\{\lambda-\frac{|\nabla u|^{2}}{2}, 0\right\} \leq \int_{B_{\rho} \cap \Omega_{u}} \max \left\{\frac{|\nabla u|^{2}}{2}-\lambda, 0\right\} \\
+\left(\lambda-\mu^{\prime}(\rho)\right) \pi \rho^{2}+\frac{C r^{2}}{\log (\rho / r)}
\end{array}
$$

As in [2], we use the (modified) Theorem 6.3 with $Q \equiv \lambda / 2$.

$$
f_{B_{r} \cap \Omega_{u}} \max \left\{\lambda-\frac{|\nabla u|^{2}}{2}, 0\right\} \leq C_{1}\left(\frac{\rho}{r}\right)^{2}\left(\frac{\rho}{R}\right)^{\alpha}+\left(\lambda-\mu^{\prime}(\rho)\right) \pi\left(\frac{\rho}{r}\right)^{2}+\frac{C}{\log (\rho / r)}
$$

For the choice of $r, \rho$ and $R$, here we can take $R=R_{0}$ constant, but we have an other term in $\lambda-\mu^{\prime}(\rho)$. We can choose $r=\rho\left(\pi\left(\lambda-\mu^{\prime}(\rho)\right)+C_{1}(\rho / R)^{\alpha}\right)^{1 / 4}<\rho$ for $\rho$ small enough (Prop. 4.3). With this choice we have:

$$
\begin{aligned}
f_{B_{r} \cap \Omega_{u}} \max \left\{\lambda-\frac{|\nabla u|^{2}}{2}, 0\right\} \leq & \frac{\pi\left(\left(\lambda-\mu^{\prime}(\rho)\right)+C_{1}(\rho / R)^{\alpha}\right)}{\pi\left(\left(\lambda-\mu^{\prime}(\rho)\right)+C_{1}(\rho / R)^{\alpha}\right)^{1 / 2}} \\
& +\frac{C}{\log \left(\pi\left(\lambda-\mu^{\prime}(\rho)\right)+C_{1}(\rho / R)^{\alpha}\right)^{1 / 4}}
\end{aligned}
$$

which goes to 0 when $\rho$ goes to 0 , using Proposition 4.3 .

\section{The hypothesis $\lambda>0$}

In this section, we discuss the hypothesis $\lambda>0$. A main result is the following.

Proposition 6.1. Let $u$ be a solution of $(\mathcal{P})$ with $f \geq 0, f \not \equiv 0$. Then we have $\lambda>0$.

Proof. This proposition comes from the more general Proposition 6.2 (where we do not make assumption on the sign of $f$ ). If $f \geq 0$, we take $D_{1}$ such that conditions (22) in Section 4 are true. By Proposition $6.2,-\Delta u \geq 0$ in $D_{1}$, so that $u>0$ in $D_{1}$. This contradicts the conditions on $D_{1}$.

Proposition 6.2. Let $u$ be a solution of $(\mathcal{P})$ such that $\lambda=0$. Let $D_{1}$ be an open subset satisfying conditions $(22)$ and such that $u \geq 0$ on $D_{1}$. Then we have that:

$$
-\Delta u=f \chi_{\Omega u} \text { in } D_{1}
$$

Proof. We will show that $-\Delta u=f \chi_{\Omega_{u}}$ in every regular open subset $D_{2}$ such that $\overline{D_{2}} \subset D_{1}$. To make the proof more clear, we will take $D_{1}=B_{3}=B\left(0,3 r_{1}\right)\left(r_{1}\right.$ small enough) and $D_{2}=B_{1}=B\left(0, r_{1}\right)$ but there is no changes for arbitrary open subset. Let $\left(\omega_{n}\right)$ be an increasing sequence of regular open subsets of $B_{3} \cap \Omega_{u}$ such that $\left(B_{2}=B\left(0,2 r_{1}\right)\right)$

$$
\left\{x \in \overline{B_{2} \cap \Omega_{u}}, d\left(x, \partial \Omega_{u}\right) \geq 1 / n\right\} \subset \omega_{n} \subset \overline{\omega_{n}} \subset B_{3} \cap \Omega_{u}
$$

(see the picture below). In particular, $B_{2} \cap \omega_{n}$ increases to $B_{2} \cap \Omega_{u}$. 


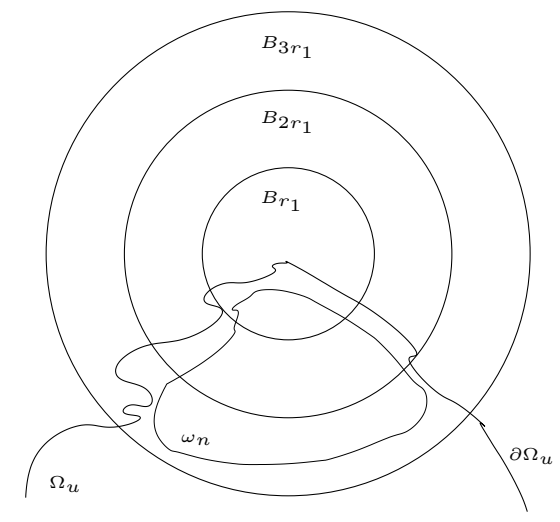

For every $\varphi \in \mathrm{C}_{0}^{\infty}\left(B_{1}\right)\left(-\Delta u=f\right.$ in $\omega_{n}$ and $\Delta u$ is a measure),

$$
\langle-\Delta u, \varphi\rangle=\int_{B_{1}} \varphi f \chi_{\omega_{n}}-\int_{B_{1} \backslash \omega_{n}} \varphi \mathrm{d}(\Delta u) .
$$

The first term goes to $\int_{B_{1}} \varphi f \chi_{\Omega_{u}}$ so we need to show that the second one converges to 0 to get $-\Delta u=f \chi_{\Omega_{u}}$ in $B_{1}$.

Let $\theta \in \mathrm{C}_{0}^{\infty}\left(B_{2}\right)$ be such that $0 \leq \theta \leq 1$ and $\theta=1$ in $\overline{B_{1}}$. Let $H_{n}$ be the vector valued function defined by:

$$
H_{n}=\left\{\begin{array}{l}
\theta \nabla u \text { on } B_{3} \backslash \omega_{n} \\
\text { harmonic on } \omega_{n}
\end{array}\right.
$$

(by harmonic, we mean that each component is harmonic). This function is continuous on the boundary of $\omega_{n}$ $\left(\nabla u\right.$ is continuous in $\left.\Omega_{u}\right)$, so $\operatorname{div} H_{n}$ does not charge $\partial \omega_{n}$ and we get, using $\theta \equiv 1$ in $B_{1}$, for every $\varphi \in \mathrm{C}_{0}^{\infty}\left(B_{1}\right)$,

$$
\left\langle\operatorname{div}\left(H_{n}\right) \cdot \varphi\right\rangle=\int_{B_{1} \cap \omega_{n}} \operatorname{div}\left(H_{n}\right) \varphi+\int_{B_{1} \backslash \omega_{n}} \varphi \mathrm{d}(\Delta u) .
$$

We want to show that the last term of this equation goes to 0 : for this we will show that the two first ones go to 0 .

More precisely, we will prove that

$$
H_{n} \text { converges uniformly to } 0 \text { on } B_{2}
$$

$$
\left\|\operatorname{div} H_{n}\right\|_{L^{2}, B_{1} \cap \omega_{n}} \leq C
$$

From (35), we deduce that the first term in (34) tends to 0 . We also deduce that, since div $H_{n}$ is harmonic in $\omega_{n}$, it converges to 0 on any compact set of $B_{2} \cap \Omega_{u}$ (recall that $B_{2} \cap \omega_{n}$ increases to $B_{2} \cap \Omega_{u}$ ). Coupled with (36) this implies that $\operatorname{div} H_{n} \chi_{B_{1} \cap \omega_{n}}$ tends to 0 in $\mathcal{D}^{\prime}\left(B_{1}\right)$ and the proof of Proposition 6.2 will be complete.

Using the maximum principle, we have that:

$$
\left\|H_{n}\right\|_{\infty, \omega_{n}} \leq\|\theta \nabla u\|_{\infty, \partial \omega_{n}} \leq\|\nabla u\|_{\infty, B_{2} \cap \partial \omega_{n}}
$$

By the following Lemma 6.3 and since $\nabla u=0$ outside $\Omega_{u}$, we deduce (35). 
We now prove (36). Let $i=1 \ldots d$, we have $H_{n}^{i}-\theta u_{x_{i}} \in \mathrm{H}_{0}^{1}\left(\omega_{n}\right)\left(H_{n}^{i}\right.$ is the $\mathrm{i}$-th component of $\left.H_{n}\right)$, taking the Laplacian, we get

$$
\Delta\left(H_{n}^{i}-\theta u_{x_{i}}\right)=\Delta\left((u \theta)_{x_{i}}-u \theta_{x_{i}}\right)=(\Delta(u \theta))_{x_{i}}-\Delta\left(u \theta_{x_{i}}\right) .
$$

Since in $\omega_{n}, \Delta(u \theta)=-f \theta+u \Delta \theta+2\langle\nabla u \cdot \nabla \theta\rangle$ and $\nabla\left(u \theta_{x_{i}}\right)=\theta_{x_{i}} \nabla u+u \nabla \theta_{x_{i}}$ are bounded functions $(u$ and $\nabla u$ are bounded), we can multiply by $H_{n}^{i}-\theta u_{x_{i}}$ and integrate by parts to have,

$$
-\left\|\nabla\left(H_{n}^{i}-\theta u_{x_{i}}\right)\right\|_{L^{2}, \omega_{n}}^{2}=-\int_{\omega_{n}} \Delta(u \theta)\left(H_{n}^{i}-\theta u_{x_{i}}\right)_{x_{i}}+\int_{\omega_{n}}\left\langle\nabla\left(u \theta_{x_{i}}\right) \cdot \nabla\left(H_{n}^{i}-\theta u_{x_{i}}\right)\right\rangle,
$$

and so,

$$
\left\|\nabla\left(H_{n}^{i}-\theta u_{x_{i}}\right)\right\|_{L^{2}, \omega_{n}}^{2} \leq\|\Delta(u \theta)\|_{L^{2}, \omega_{n}}\left\|\nabla\left(H_{n}^{i}-\theta u_{x_{i}}\right)\right\|_{L^{2}, \omega_{n}}+\left\|\nabla\left(u \theta_{x_{i}}\right)\right\|_{L^{2}, \omega_{n}}\left\|\nabla\left(H_{n}^{i}-\theta u_{x_{i}}\right)\right\|_{L^{2}, \omega_{n}} .
$$

This implies that $\left\|\nabla\left(H_{n}^{i}-\theta u_{x_{i}}\right)\right\|_{L^{2}, \omega_{n}}$ is bounded. Since

$$
\operatorname{div} H_{n}=\sum_{i=1}^{d}\left(\left(H_{n}^{i}-\theta u_{x_{i}}\right)_{x_{i}}+\theta_{x_{i}} u_{x_{i}}+\theta u_{x_{i} x_{i}}\right)
$$

using that $-\Delta u=f$ in $\omega_{n}$, we get that $\operatorname{div} H_{n}$ is bounded in $L^{2}\left(\omega_{n}\right)$.

It remains to prove the following lemma.

Lemma 6.3. Using the same notations as in the previous proposition, we have that:

$$
\lim _{n \rightarrow \infty}\|\nabla u\|_{\infty, B_{2} \cap\left(\Omega_{u} \backslash \omega_{n}\right)}=0 .
$$

Proof. We will first show that there exists a decreasing function $\eta$ with $\lim _{r \rightarrow 0} \eta(r)=0$, such that if $x_{0} \in B_{2}$, $0 \leq r \leq 1$ and $\left|\{u=0\} \cap B\left(x_{0}, r\right)\right|>0$ then,

$$
\frac{1}{r} f_{\partial B\left(x_{0}, r\right)} u \leq \eta(r)
$$

Let $B_{r}=B\left(x_{0}, r\right)$ be such a ball. Using exactly the same computation as in Proposition 2.5 in [10], we define $v$ by $-\Delta v=f$ in $B_{r}$ and $v=u$ outside $B_{r}$ and we have $\left(B_{r} \subset D_{1}=B(0,3)\right)$ :

$$
\left.\int_{B_{r}}|\nabla(u-v)|^{2} \leq \lambda^{*}\left(\omega_{d} r^{d}\right) \mid\{u=0\} \cap B_{r}\right\} \mid,
$$

(see Prop. 4.3 for the definition of $\lambda^{*}$ and Rem. 4.6). We also get as in [2,10],

$$
\left.\mid\{u=0\} \cap B_{r}\right\}\left.\left|\left(\frac{1}{r} f_{\partial B_{r}} u\right)^{2} \leq C \int_{B_{r}}\right| \nabla(u-v)\right|^{2} .
$$

Actually, this is true only if $\frac{1}{r} f_{\partial B_{r}} u-C r \geq 0$ where $C$ depends only on $d$ and $f$, but otherwise $\eta(r) \geq C r$ works. With (38) and (39), we get (37) with $\eta(r)=C \sqrt{\lambda^{*}\left(\omega_{d} r^{d}\right)}+C r$. (Using Rem. 4.6, $\lambda^{*}$ is decreasing and $\lim _{h \rightarrow 0} \lambda^{*}(h)=0$.)

Let $x_{0} \in\left(\Omega_{u} \backslash \omega_{n}\right) \cap B_{2}$ and $B_{r_{0}}=B\left(x_{0}, r\right)$ be the largest ball included in $\Omega_{u}$. By definition of $\omega_{n}$, we have $r_{0} \leq 1 / n($ see $(32))$. 
For $\delta>0$ we have that $\{u=0\} \cap B_{r_{0}+\delta} \neq \emptyset$. So there are two cases.

1) (Good case.) If $\left|\{u=0\} \cap B_{r+\delta_{0}}=0\right|$ for some $\delta_{0}$ then we see from (38) that $-\Delta u=f$ in $B_{r_{0}+\delta_{0}}$. Let $0<\delta<\delta_{0}$ and $x \in B_{r_{0}+\delta}$ be such that $u(x)=0$, now we use Lemma 2.4 in [10] on $B_{r_{0}+\delta}$ in $x$ and we get $(C$ depends only on $d$ and $f$ ):

$$
\frac{1}{r_{0}+\delta} f_{\partial B_{r_{0}+\delta}} u \leq C\left(r_{0}+\delta\right) \leq \eta\left(2 r_{0}\right)
$$

And when $\delta$ goes to 0 :

$$
\frac{1}{r_{0}} f_{\partial B_{r_{0}}} u \leq \eta\left(2 r_{0}\right)
$$

2) If for every $0<\delta<r_{0}\left|\{u=0\} \cap B_{r+\delta}\right|>0$ we can use (37),

$$
\frac{1}{r_{0}+\delta} f_{\partial B_{r_{0}+\delta}} u \leq \eta\left(r_{0}+\delta\right) \leq \eta\left(2 r_{0}\right)
$$

and, when $\delta$ goes to 0 ,

Now we use that

$$
\frac{1}{r_{0}} f_{\partial B_{r_{0}}} u \leq \eta\left(2 r_{0}\right)
$$

$$
\left|\nabla u\left(x_{0}\right)\right| \leq C \frac{1}{r_{0}} f_{\partial B_{r_{0}}} u+C r_{0}
$$

(see Prop. 2.4 in [10], in $B_{r_{0}}$ we have $-\Delta u=f$ ). Using this in (41) or in (40) we have $\left(r_{0} \leq \frac{1}{n}\right)$

$$
\left|\nabla u\left(x_{0}\right)\right| \leq \eta(2 / n)+C / n,
$$

which goes to 0 when $n$ goes to infinity.

Remark 6.4. By moving $D_{1}$ in all $D$ in Proposition 6.2, we could see that, if $\lambda=0$ then

$$
-\Delta u=f \chi_{\Omega u} \text { in } D .
$$

We can compare this with the hypothesis (7) taken at the beginning of the paper which excludes existence of $w$ such that $-\Delta w=f$ in $D$ and $\left|\Omega_{w}\right| \leq a$.

Remark 6.5. It is likely that a result similar to Proposition 6.2 is true when $u$ changes its sign, but the proof of Lemma 6.3 strongly uses that $u \geq 0$. Let us mention that, if (7) is true, we can prove that the set of $b$ for which $\lambda_{u_{b}}>0$ is a dense open set in $(0, a)$ where $u_{b}$ is any solution of

$$
J\left(u_{b}\right)=\min \left\{J(u), u \in \mathrm{H}^{1}(D),\left|\Omega_{u}\right|=b\right\} .
$$

\section{REFERENCES}

[1] N. Aguilera, H.W. Alt and L.A. Caffarelli, An optimization problem with volume constraint. SIAM J. Control Optimization 24 (1986) 191-198.

[2] H.W. Alt and L.A. Caffarelli, Existence and regularity for a minimum problem with free boundary. J. Reine Angew. Math. 325 (1981) 105-144.

[3] H.W. Alt, L.A. Caffarelli and A. Friedman, Variational problems with two phases and their free boundaries. Trans. Am. Math. Soc. 282 (1984) 431-461.

[4] T. Briancon, Problèmes de régularité en optimisation de formes. Ph.D. thesis, université Rennes 1 (2002).

[5] M. Crouzeix, Variational approach of a magnetic shaping problem. Eur. J. Mech. 10 (1991) 527-536.

[6] L.C. Evans and R.F. Gariepy, Measure theory and fine properties of functions. CRC Press (1992).

[7] H. Federer, Geometric measure theory. Springer-Verlag (1969). 
[8] D. Gilbarg and N.S. Trudinger, Elliptic Partial Differential Equations of Second Order. Springer-Verlag (1983).

[9] E. Giusti, Minimal surfaces and functions of bounded variation. Birkhäuser (1986).

[10] B. Gustafsson and H. Shahgholian, Existence and geometric properties of solutions of a free boundary problem in potential theory. J. Reine Angew. Math. 473 (1996) 137-179.

[11] M. Hayouni, Existence et régularité pour des problèmes d'optimisation de formes. Ph.D. thesis, université Henri Poincaré Nancy 1 (1997).

[12] M. Hayouni, Lipschitz continuity of the state function in a shape optimization problem. J. Convex Anal. 6 (1999) 71-90.

[13] M. Hayouni, T. Briancon and M. Pierre. On a volume constrained shape optimization problem with nonlinear state equation. (to appear).

[14] X. Pelgrin, Étude d'un problème à frontière libre bidimensionnel. Ph.D. thesis, université Rennes 1 (1994).

[15] T.H. Wolff, Plane harmonic measures live on sets of $\sigma$-finite length. Ark. Mat. 31 (1993) 137-172. 